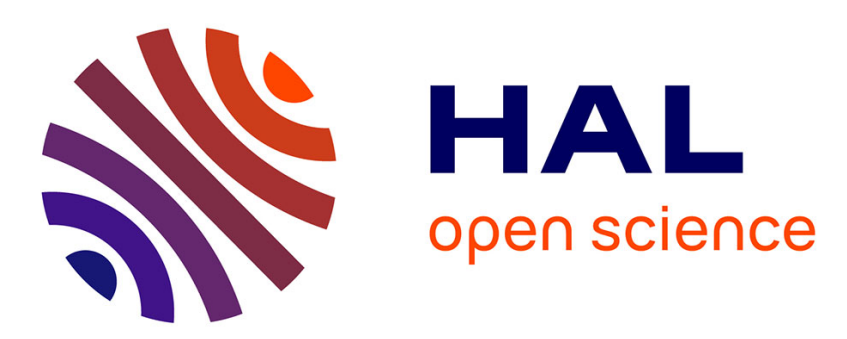

\title{
On the Factors Affecting the Accuracy and Robustness of Smoothed-Radial Point Interpolation Method
} Abderrachid Hamrani, Idir Belaidi, Eric Monteiro, Philippe Lorong

\section{To cite this version:}

Abderrachid Hamrani, Idir Belaidi, Eric Monteiro, Philippe Lorong. On the Factors Affecting the Accuracy and Robustness of Smoothed-Radial Point Interpolation Method. Advances in Applied Mathematics and Mechanics, 2017, 9 (1), pp.43-72. 10.4208/aamm.2015.m1115 . hal-01559519

\section{HAL Id: hal-01559519 https://hal.science/hal-01559519}

Submitted on 10 Jul 2017

HAL is a multi-disciplinary open access archive for the deposit and dissemination of scientific research documents, whether they are published or not. The documents may come from teaching and research institutions in France or abroad, or from public or private research centers.
L'archive ouverte pluridisciplinaire HAL, est destinée au dépôt et à la diffusion de documents scientifiques de niveau recherche, publiés ou non, émanant des établissements d'enseignement et de recherche français ou étrangers, des laboratoires publics ou privés. 


\title{
On the Factors Affecting the Accuracy and Robustness of Smoothed-Radial Point Interpolation Method
}

\author{
Abderrachid Hamrani ${ }^{1,2, *}$, Idir Belaidi ${ }^{1}$, Eric Monteiro ${ }^{2}$ and \\ Philippe Lorong ${ }^{2}$ \\ ${ }^{1}$ Research team MISP, LEMI, University of M'Hamed Bougara de Boumerdes, 35000, \\ Algérie \\ 2 PIMM, Arts et Métiers ParisTech, CNRS, 151 bd de l'Hôpital, 75013 Paris, France
}

Received 17 May 2015; Accepted (in revised version) 8 December 2015

\begin{abstract}
In order to overcome the possible singularity associated with the Point Interpolation Method (PIM), the Radial Point Interpolation Method (RPIM) was proposed by G. R. Liu. Radial basis functions (RBF) was used in RPIM as basis functions for interpolation. All these radial basis functions include shape parameters. The choice of these shape parameters has been and stays a problematic theme in RBF approximation and interpolation theory. The object of this study is to contribute to the analysis of how these shape parameters affect the accuracy of the radial PIM. The RPIM is studied based on the global Galerkin weak form performed using two integration technics: classical Gaussian integration and the strain smoothing integration scheme. The numerical performance of this method is tested on their behavior on curve fitting, and on three elastic mechanical problems with regular or irregular nodes distributions. A range of recommended shape parameters is obtained from the analysis of different error indexes and also the condition number of the matrix system. All resulting RPIM methods perform very well in term of numerical computation. The Smoothed Radial Point Interpolation Method (SRPIM) shows a higher accuracy, especially in a situation of distorted node scheme.
\end{abstract}

AMS subject classifications: 65N12, 97M50

Key words: Radial Basis Function, Radial Point Interpolation Methods, strain smoothing nodal integration, Galerkin weak form.

\section{Introduction}

One of most developed numerical techniques is the finite element method (FEM). In the FEM, a continuum solid is divided into a set of finite elements, the mesh of the solid,

*Corresponding author.

Email: hamrani.abderrachid@gmail.com (A. Hamrani), idir.belaidi@gmail.com (I. Belaidi), eric.monteiro@ ensam.eu (E. Monteiro), philippe.lorong@ensam.eu (P. Lorong) 
which are connected between them by nodes. The FEM proved to be effective and robust in several engineering fields because of its capacity in dealing with complex geometries. However, this method suffers from some limitations when severe element distortions take place under large deformation processes. In this context, the accuracy of results are lost [1]. To surmount this problem, meshless or meshfree methods are proposed where the problem domain is represented by a set of scattered nodes, without the need of any information about relationship between them. The development of some of the meshless methods goes back more than seventy years, with the appearance of collocation methods [3-5]. After that, the first meshless method known as the Smoothed Particle Hydrodynamics (SPH) [6], was originally used for the simulation of astrophysical phenomena by Lucy [6,7]. From early 1990s, numerous methods have been proposed; for instance the diffuse element method (DEM) [8], the reproducing kernel particle method (RKPM) [9,10],the element free Galerkin (EFG) method [11], the point interpolation methods [12], the meshless local Petrov-Galerkin method (MLPG) [13]. All these methods use meshless shape functions to represent the field variables, since these shape functions are mathematically constructed by using only a set of nodes without requiring a mesh. The Moving least square (MLS) interpolation was one of the first shape functions used by Belytschko et al. [14] for the development of the element free Galerkin (EFG) method. Because some of its limitations, in particular, the complexity of the calculations of MLS shape functions and their partial derivatives, besides the difficulty of imposing boundary conditions [15]. Liu and $\mathrm{Gu}[16,17]$ proposed a new family of meshless shape functions: "Point Interpolation Methods". Among these methods, the radial point interpolation method (RPIM), is preferred because the use of radial basis function avoids the problem of singularity such as conventional PIM $[18,55]$. The shape functions resulting from RBF are stable and hence flexible for arbitrary and irregular nodal configurations. For numerical simulations of mechanics problems it is needed to combine shape function with formulation procedure based on strong or weak-forms derived directly from the physics. In meshless collocation methods (based on the strong-form), the PDE is usually discretized at nodes by some forms of collocation, therefore no background cells are required for numerical integration. There are various meshless based strong-form methods, e.g., the Finite Point Method (FPM) [49-51], the HP-Meshless Cloud Method [56] and the Radial Basis Function Collocation Methods (RBF-CM) [57-60], etc. In this paper, Galerkin weak form is used to construct discretized system equations. For the requirement of a weaker consistency on the approximate function, weak forms need an integral operation performed numerically by the use of two major techniques: the classical Gauss integration and the stabilized conforming nodal integration (SCNI) proposed by Beissel and Belytschko [19] and later by Chen et al. [20,21]. The objective of the present work, is to study the RBF meshfree Galerkin Methods through their performances in term of: interpolations (RPIM shape function) and numerical integration techniques (classical Gauss integration and strain smoothing nodal integration). The paper is organized as follows. Firstly, an introduction on the used RBF shape functions and the integration schemes is given. A short outline of the algorithm used for the analysis is presented. After that, the 
results of the numerical study on the performance of all variants of the RPIM method (analysis of all RBF shape functions and the two integration schemes) are given. Afterwards the computational efficiency of the RPIM methods is compared. The conclusion is given in the last section.

\section{Radial Basis Functions (RBF)}

Let be a function $\mathbf{R}: \mathbb{R}^{d} \rightarrow \mathbb{R}$ supposed to be conditionally positive definite of order $\tau$ [44], and $X=\left\{x_{1}, \cdots, x_{N}\right\} \subseteq \mathbb{R}^{d}$ consisting of pairwise distinct centers $x_{i}$. The radial basis function interpolant $S_{u}$ to a function $u \in C\left(\mathbb{R}^{d}\right)$ on a set of centers $X$ is given by

$$
S_{u}(x)=\sum_{i=1}^{n} a_{i} R\left(\mathbf{x}-\mathbf{x}_{i}\right)+p(\mathbf{x}),
$$

where $p$ is a polynomial of degree less than the order of RBF $\tau$. By interpolation, $S_{u}$ has to satisfy $S_{u}\left(\mathbf{x}_{i}\right)=u\left(\mathbf{x}_{i}\right), 1 \leq i \leq n$. The kind of RBFs, we will be mostly interested in are:

1. RBFs with global support: Multiquadric (MQ), Gaussian (EXP), Piecewise polynomial $(\mathrm{Pp})\left(r_{i}=r\left(\mathbf{x}-\mathbf{x}_{i}\right)=\sqrt{\left(x-x_{i}\right)^{2}+\left(y-y_{i}\right)^{2}}\right.$ in 2D and $d_{c}$ is the size of the support domain) (Table 1$)$.

Table 1: Radial Basis Functions with global support used in this study.

\begin{tabular}{||c|c|c|c||}
\hline RBF & Expression & Parameters & Order \\
\hline MQ [46] & $R_{i}(\mathbf{x})=\left(r_{i}^{2}+\left(\alpha_{c} d_{c}\right)^{2}\right)^{q}$ & $\alpha_{c} \in \mathbb{R}_{\geq 0}, q \in \mathbb{R}_{>0} \backslash\{0,1,2, \cdots\}$ & $\lceil q\rceil$ \\
EXP [46] & $R_{i}(\mathbf{x})=\exp \left[-\alpha_{c}\left(\frac{r_{i}}{d_{c}}\right)^{2}\right]$ & $\alpha_{c} \in \mathbb{R}_{>0}$ & 0 \\
$\operatorname{Pp}[48]$ & $R_{i}(\mathbf{x})=r_{i}^{\eta}$ & $\eta \in \mathbb{R}_{>0} \backslash 2 \mathbb{N}$ & $\left\lceil\frac{\eta}{2}\right\rceil$ \\
\hline
\end{tabular}

2. RBF with compact support: (Wu-C2) [45] (Eq. (2.2)) $\left(\delta_{c}\right.$ is the size of the local compact support):

$$
R_{i}(\mathbf{x})=\left(1-\frac{r_{i}}{\delta_{c}}\right)^{5}\left(8+40 \frac{r_{i}}{\delta_{c}}+48 \frac{r_{i}{ }^{2}}{\delta_{c}^{2}}+25 \frac{r_{i}{ }^{3}}{\delta_{c}^{3}}+5 \frac{r_{i}{ }^{4}}{\delta_{c}^{4}}\right) .
$$

\section{Construction of RPIM shape functions}

The construction of RBF shape functions augmented with polynomials is based on the Eq. (2.1), which can be rewritten as follow [55]:

$$
u(\mathbf{x})=\sum_{i=1}^{n} R_{i}(\mathbf{x}) a_{i}+\sum_{j=1}^{m} p_{j}(\mathbf{x}) b_{j}=\mathbf{R}^{T}(\mathbf{x}) \mathbf{a}+\mathbf{p}^{T}(\mathbf{x}) \mathbf{b},
$$


where $R_{i}(\mathbf{x})$ is a radial basis function $(\mathrm{RBF}), p_{j}(\mathbf{x})$ is a basis function of monomials in the space coordinates (for example $\mathbf{p}^{T}$ can be $[1, x, y]$ in 2D problems). Coefficients $a_{i}$ and $b_{i}$ represent constants to be determined, $n$ the number of RBFs and $m$ the number of polynomial basis functions. The coefficients $a_{i}$ et $b_{i}$ must satisfy Eq. (3.1) at $n$ nodes in the local support domain of the point of interest $\mathbf{x}$. This leads to $n$ linear equations, the matrix form of these equations can be written as:

$$
\mathbf{u}=\mathbf{R}_{0} \mathbf{a}+\mathbf{P}_{m} \mathbf{b},
$$

where

$$
\mathbf{u}=\left\{\begin{array}{llll}
u_{1} & u_{2} & \cdots & u_{n}
\end{array}\right\}^{T}, \quad \mathbf{a}^{T}=\left\{\begin{array}{llll}
a_{1} & a_{2} & \cdots & a_{n}
\end{array}\right\} ， \mathbf{b}^{T}=\left\{\begin{array}{llll}
b_{1} & b_{2} & \cdots & b_{m}
\end{array}\right\},
$$

and the RBF matrix

$$
\mathbf{R}_{0}=\left[\begin{array}{cccc}
R_{1}\left(\mathbf{x}_{1}\right) & R_{2}\left(\mathbf{x}_{1}\right) & \cdots & R_{n}\left(\mathbf{x}_{1}\right) \\
R_{1}\left(\mathbf{x}_{2}\right) & R_{2}\left(\mathbf{x}_{2}\right) & \cdots & R_{n}\left(\mathbf{x}_{2}\right) \\
\cdots & \cdots & \cdots & \cdots \\
R_{1}\left(\mathbf{x}_{n}\right) & R_{2}\left(\mathbf{x}_{n}\right) & \cdots & R_{n}\left(\mathbf{x}_{n}\right)
\end{array}\right]_{(n \times n)} .
$$

The Polynomial basis functions matrix:

$$
\mathbf{P}_{m}=\left[\begin{array}{ccccc}
1 & x_{1} & y_{1} & \cdots & p_{m}\left(\mathbf{x}_{1}\right) \\
1 & x_{2} & y_{2} & \cdots & p_{m}\left(\mathbf{x}_{2}\right) \\
\vdots & \vdots & \vdots & \ddots & \vdots \\
1 & x_{n} & y_{n} & \cdots & p_{m}\left(\mathbf{x}_{n}\right)
\end{array}\right]
$$

There are $n+m$ variables in Eq. (3.2), so another $m$ equations are required. Golberg et al. [22] added additional $m$ equations by using the following constraint conditions

$$
\mathbf{P}_{m}^{T} \mathbf{a}=\sum_{i=1}^{n} p_{j}\left(\mathbf{x}_{i}\right) a_{i}=0, \quad j=1,2, \cdots, m .
$$

Eqs. (3.2) and (3.6) can be written in the following form

$$
\begin{aligned}
& \tilde{\boldsymbol{u}}=\left[\begin{array}{l}
\mathbf{u} \\
0
\end{array}\right]=\underbrace{\left[\begin{array}{cccc}
\mathbf{R}_{0} & \mathbf{P}_{m} \\
\mathbf{P}_{m}^{T} & 0
\end{array}\right]}_{\mathbf{G}}\left\{\begin{array}{l}
\mathbf{a} \\
\mathbf{b}
\end{array}\right\}=\mathbf{G a}_{0}, \\
& \mathbf{a}_{0}^{T}=\left\{\begin{array}{llllllll}
a_{1} & a_{2} & \cdots & a_{n} & b_{1} & b_{2} & \cdots & b_{m}
\end{array}\right\}, \\
& \tilde{\boldsymbol{u}}^{T}=\left\{\begin{array}{llllllll}
u_{1} & u_{2} & \cdots & u_{n} & 0 & 0 & \cdots & 0
\end{array}\right\} .
\end{aligned}
$$

Since the matrix $\mathbf{R}_{0}$ is symmetric, matrix $\mathbf{G}$ is also symmetric, then by solving Eq. (3.7a), we obtain

$$
\mathbf{a}_{0}=\left\{\begin{array}{l}
\mathbf{a} \\
\mathbf{b}
\end{array}\right\}=\mathbf{G}^{-1} \tilde{\boldsymbol{u}}
$$


To ensure that the inverse of the moment matrix $\mathrm{G}$ is well-defined, the polynomial base added to the RBF interpolation cannot be arbitrary [46]. A low-degree polynomial is needed to guarantee the non-singularity of the matrix $\mathrm{G}$ [47]. The RPIM shape function is finally expressed as

$$
\begin{aligned}
& \mathbf{u}(\mathbf{x})=\left\{\begin{array}{ll}
\mathbf{r}^{T}(\mathbf{x}) & \mathbf{p}^{T}(\mathbf{x})
\end{array}\right\}\left\{\begin{array}{l}
\mathbf{a} \\
\mathbf{b}
\end{array}\right\}, \\
& \mathbf{u}(\mathbf{x})=\left\{\begin{array}{lll}
\mathbf{r}^{T}(\mathbf{x}) & \mathbf{p}^{T}(\mathbf{x})
\end{array}\right\} \mathbf{G}^{-1} \tilde{\boldsymbol{u}}=\tilde{\boldsymbol{\phi}}^{T}(\mathbf{x}) \tilde{\boldsymbol{u}}, \\
& \tilde{\boldsymbol{\phi}}^{T}(\mathbf{x})=\left\{\begin{array}{llll}
\mathbf{r}^{T}(\mathbf{x}) & \mathbf{p}^{T}(\mathbf{x})
\end{array}\right\} \mathbf{G}^{-1}, \\
& \tilde{\boldsymbol{\phi}}^{T}(\mathbf{x})=\left\{\begin{array}{lllll}
\phi_{1}(\mathbf{x}) & \phi_{2}(\mathbf{x}) & \cdots & \phi_{n}(\mathbf{x}) & \phi_{n+m}(\mathbf{x})
\end{array}\right\},
\end{aligned}
$$

where the RPIM shape functions corresponding to the nodal displacements are given by:

$$
\boldsymbol{\phi}^{T}(\mathbf{x})=\left\{\begin{array}{llll}
\phi_{1}(\mathbf{x}) & \phi_{2}(\mathbf{x}) & \cdots & \phi_{n}(\mathbf{x})
\end{array}\right\} .
$$

Direct inversion of $\mathbf{G}$ can be avoided using this procedure

$$
\begin{aligned}
& \tilde{\boldsymbol{\phi}}^{T}(\mathbf{x})=\left\{\begin{array}{ll}
\mathbf{r}^{T}(\mathbf{x}) & \mathbf{p}^{T}(\mathbf{x})
\end{array}\right\} \mathbf{G}^{-1} \\
& \Rightarrow \tilde{\boldsymbol{\phi}}^{T}(\mathbf{x}) \mathbf{G}=\left\{\begin{array}{ll}
\mathbf{r}^{T}(\mathbf{x}) & \mathbf{p}^{T}(\mathbf{x})
\end{array}\right\} \\
& \Rightarrow \mathbf{G}^{T} \tilde{\boldsymbol{\phi}}=\left\{\begin{array}{l}
\mathbf{r}(\mathbf{x}) \\
\mathbf{p}(\mathbf{x})
\end{array}\right\} \text {. }
\end{aligned}
$$

By solving Eq. (3.11) using a standard linear equation solver, we can obtain RPIM shape functions at point $\mathbf{x}$ directly without computing $\mathbf{G}^{-1}$. The subsequent RPIM shape function has the delta Kronecker and partition of unity properties, and due to the addition of polynomial basis, they also fulfill the reproducing properties [55]. In this work, different radial basis functions augmented with linear polynomial basis are used to construct the present RPIM shape function, the choice of RBFs shape parameters is studied. In order to minimize the computation time, needed for the interpolation calculation at each point $\mathbf{x}$, an influence domain is associated to each radial basis function. Outside its influence domain the radial basis function is taken null. Then at a given point $\mathbf{x}$ only a subset of radial basis functions have a non null value. It is thus possible to use inside equation (3.1) a sum over this subset of RBF and to have a number $n(\mathbf{x})$ depending on $\mathbf{x}$ quite smaller than $n$. This conducts to a matrix $\mathbf{G}$ depending on $\mathbf{x}$ too but having a size $(n(\mathbf{x})+m) \cdot(n(\mathbf{x})+m)$ smaller than $(n+m) \cdot(n+m)$. For the Wu-C2 RBF the influence domain is a disc centered at $\mathbf{x}_{i}$ having $\delta_{c}$ for radius. For MQ, EXP and Pp influence domains are generally also discs centered at $\mathbf{x}_{i}$ whose radius is chosen to contain a sufficient amount of other RBF centers. The choice of the shape and the size on the influence domains will be discuss later in the paper (Section 7.1.1). The procedure used to compute RPIM shape function is prescribed by the following pseudo-code: 


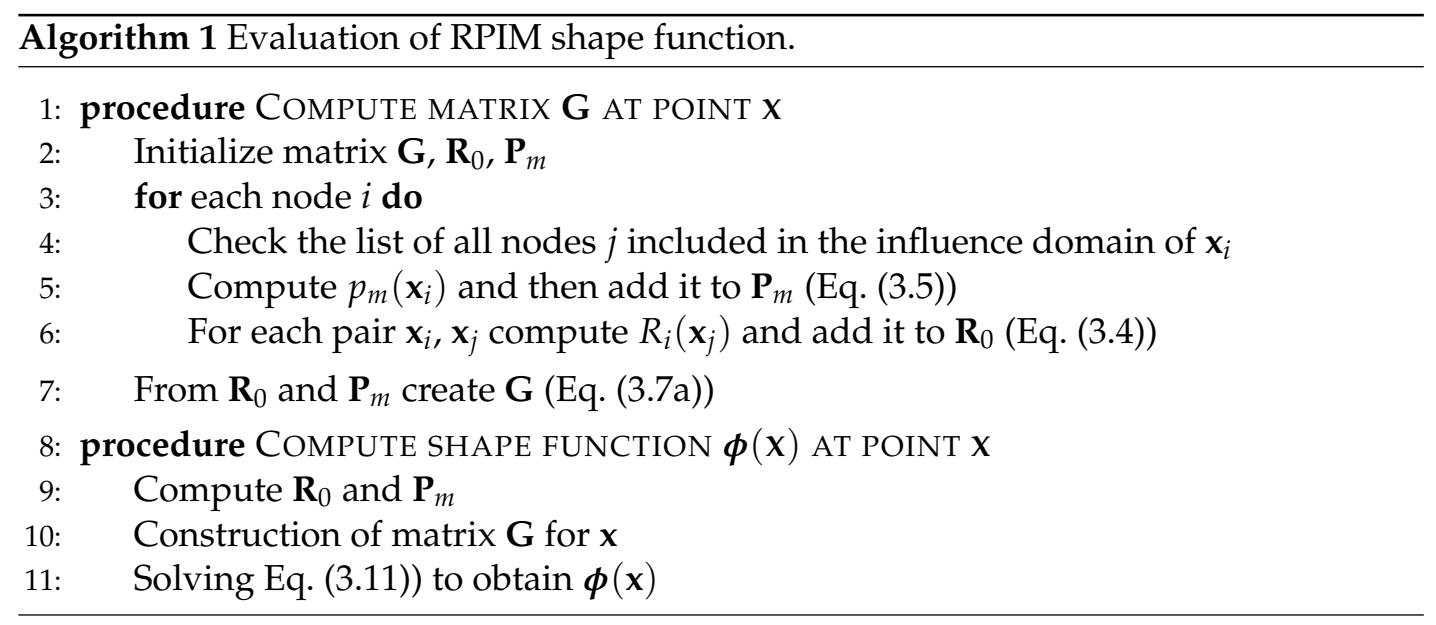

\section{Galerkin weak form of 2-D solid mechanics}

A 2-D solid mechanics static problem, defined in the domain $\Omega$ bounded by $\Gamma$, can be described by the following equilibrium equation:

$$
\nabla \cdot \sigma+\mathbf{F}=0 \text { in } \Omega \text {, }
$$

where $\sigma$ is the Cauchy stress tensor and $\mathbf{F}$ the body forces vector. The boundary conditions for the equilibrium equations are:

$$
\begin{array}{ll}
\sigma \mathbf{n}=\overline{\mathbf{t}} & \text { on the natural boundary } \Gamma_{t}, \\
\mathbf{u}=\overline{\mathbf{u}} & \text { on the essential boundary } \Gamma_{u},
\end{array}
$$

where $\overline{\mathbf{u}}$ is a prescribed displacement on boundary $\Gamma_{u}, \overline{\mathbf{t}}$ a prescribed traction on the boundary $\Gamma_{t}$ and $\mathbf{n}$ is the outward unit normal to the boundary. The well-known Galerkin weak form is given by [55]:

$$
\int_{\Omega}\left(\nabla \delta \mathbf{u}^{T}\right) \cdot \sigma d \Omega-\int_{\Omega} \delta \mathbf{u}^{T} \cdot \mathbf{F} d \Omega-\int_{\Gamma_{t}} \delta \mathbf{u}^{T} \cdot \overline{\mathbf{t}} d \Gamma=0,
$$

where $\delta$ express the variation between two states. Discretization of Eq. (4.3) with RPIM shape function given by Eq. (3.9b) yields

$$
\mathbf{K u}=\mathbf{f} \text {, }
$$

where

$$
\mathbf{K}_{i j}=\int_{\Omega} \mathbf{B}_{i}^{T} \mathbf{C} \mathbf{B}_{j} d \Omega \quad \text { and } \quad \mathbf{f}_{i}=\int_{\Omega} \boldsymbol{\Phi}_{i}^{T} \mathbf{F} d \Omega+\int_{\Gamma_{t}} \boldsymbol{\Phi}_{i}^{T} \overline{\mathbf{t}} d \Gamma,
$$


and where (in $2 \mathrm{D}$ problems) the strain matrix

$$
\mathbf{B}_{(3 \times 2 n)}=\left[\begin{array}{ccccc}
\frac{\partial \phi_{1}}{\partial x} & 0 & \cdots & \frac{\partial \phi_{n}}{\partial x} & 0 \\
0 & \frac{\partial \phi_{1}}{\partial y} & \cdots & 0 & \frac{\partial \phi_{n}}{\partial y} \\
\frac{\partial \phi_{1}}{\partial y} & \frac{\partial \phi_{1}}{\partial x} & \cdots & \frac{\partial \phi_{n}}{\partial y} & \frac{\partial \phi_{n}}{\partial x}
\end{array}\right]
$$

and $\mathbf{C}$ is the matrix of elastic constants used in FEM. For further and detailed mathematical theorems on the meshless Galerkin formulations using radial basis functions, the interested reader can refer to the work of Wendland [44].

\section{Integration techniques}

The integrals involved in Eq. (4.5) are usually evaluated numerically through the well known Gauss integration scheme as commonly used in FEM [38]. In this study, a second scheme is also used: the strain smoothing integration technique, also named as the stabilized conforming nodal integration scheme (SCNI) [53]. Both integration schemes are shortly presented below.

\subsection{Gauss integration}

In order to evaluate the integrals over the global problem domain $\Omega$ and the global boundary $\Gamma_{t}$, the problem domain is discretized into a set of background cells. Hence, the global integration can be expressed as:

$$
\int_{\Omega} \mathbf{G} d \Omega=\sum_{k=1}^{n_{c}} \int_{\Omega_{k}} \mathbf{G} d \Omega=\sum_{k=1}^{n_{c}} \sum_{i=1}^{n_{g}} w_{i} \mathbf{G}\left(\mathbf{x}_{Q i}\right)\left|\mathbf{J}_{i k}^{D}\right|,
$$

where $n_{c}$ is the number of background cells, $n_{g}$ is the number of Gauss points used in a background cell, G represents the integrand, $\Omega_{k}$ is the domain of $k$-th background cell, $w_{i}$ is the Gauss weighting factor for $i$-th Gauss point at $\mathbf{x}_{Q i}$, and $\mathbf{J}_{i k}^{D}$ is the Jacobian matrix for the area integration of the background cell $k$. The Gauss quadrature formulation on curve is obtained similarly as

$$
\int_{\Gamma_{t}} \mathbf{G} d \Gamma=\sum_{l=1}^{n_{c t}} \int_{\Gamma_{t l}} \mathbf{G} d \Gamma=\sum_{l=1}^{n_{c t}} \sum_{i=1}^{n_{g t}} \widehat{w}_{i} \mathbf{G}\left(\mathbf{x}_{Q t i}\right)\left|\mathbf{J}_{i l}^{B}\right|
$$

$n_{c t}$ is the number of curve cells used on the boundary $\Gamma_{t}$, and $n_{g t}$ is number of Gauss points used in a sub-curve, $\mathbf{J}_{i l}^{B}$ is the Jacobian matrix for curve integration of the subboundary $l$ for the Gauss point at $\mathbf{x}_{\mathrm{Q} t i}$. 


\subsection{Nodal integration}

In Gauss quadrature a global background cell structure has to be used, this fact made the method not truly meshless. To avoid the use of background cells Beissel and Belytschko [19] have proposed a nodal integration procedure based on a strain smoothing stabilization. This technique of integration is based on the substitution of the displacement gradient at a node $\mathbf{x}_{k}$ by averaging the displacement gradient over a cell accompanying that node [20]:

$$
\nabla \mathbf{u}^{h}(\mathbf{x})=\int_{\Omega_{k}} \nabla \mathbf{u}^{h}(\mathbf{x}) \tilde{W}\left(\mathbf{x}_{k}-\mathbf{x}\right) d \Omega,
$$

$\tilde{W}\left(\mathbf{x}_{k}-\mathbf{x}\right)$ is a smoothing or weight function associated with $\mathbf{x}_{k}$. In general the following simplest form of the Heaviside-type smoothing function is used:

$$
\widetilde{W}\left(\mathbf{x}_{k}-\mathbf{x}\right)= \begin{cases}1 / A_{k}, & \mathbf{x} \in \Omega_{k}, \\ 0, & \mathbf{x} \notin \Omega_{k}\end{cases}
$$

where $A_{k}$ is the area of smoothing domain $\Omega_{k}$, then Eq. (5.3) becomes:

$$
\nabla \mathbf{u}^{h}(\mathbf{x})=\frac{1}{A_{k}} \int_{\Omega_{k}} \nabla \mathbf{u}^{h}(\mathbf{x}) d \Omega
$$

The surface (or volume) integral can be rewritten by means of Gauss divergence theorem to a curve (surface) integral:

$$
\widetilde{\nabla} \mathbf{u}^{h}(\mathbf{x})=\int_{\Gamma_{k}} \mathbf{u}^{h}(\mathbf{x}) \mathbf{n}_{k}(\mathbf{x}) \tilde{W}\left(\mathbf{x}_{k}-\mathbf{x}\right) d \Gamma=\frac{1}{A_{k}} \int_{\Gamma_{k}} \mathbf{u}^{h}(\mathbf{x}) \mathbf{n}_{k}(\mathbf{x}) d \Gamma .
$$

The strain/gradient smoothing integration technique is principaly used on the smoothed finite element methods (SFEM) [35-37,39]. Numbers of SFEM models was developed, depending on the types of smoothing domains used [53]. Different smoothing domains created based on cells (cell-based S-FEM (CS-FEM)) [40], nodes (node-based S-FEM (NSFEM)) [43], edges (edge-based S-FEM (ES-FEM) [42], and faces (face-based S-FEM (FSFEM) [41]. The usage of the SCNI technique with the RPIM conducts to the S-RPIM, the Smoothed Radial Point Interpolation Method.

\section{RPIM program description}

The algorithm of the RPIM source code implemented in Matlab, is shown in Algorithm 2. The procedure is as follow:

Step 1 When the geometry of the problem domain is created, a set of field nodes is generated to represent the problem domain.

Step 2 The global background cells are built for numerical integrations. 
Step 3 The system matrices are assembled through two loops: the outer loop is for all the quadrature cells, and the inner loop is for all the Gauss quadrature points in a cell.

Step 4 The boundary conditions are enforced.

Step 5 The system equation is solved using the standard equation solvers.

Step 6 The post-processing is performed to obtain the results in term of displacements and stresses.

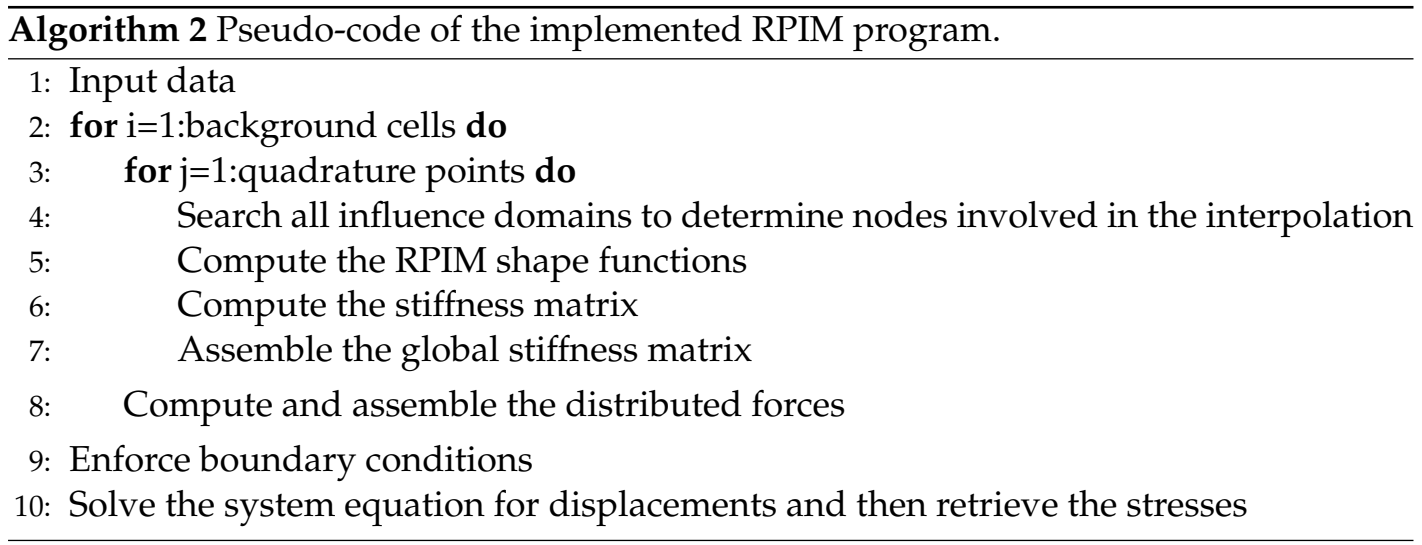

\section{Error analysis for Radial Point Interpolation Method}

Two sources of error are noticed in case of RPIM: the Radial Basis Function interpolation error and the error in calculation of Galerkin weak form. The first error due to interpolation is evaluated by the error in fitting different surfaces. The second error is evaluated through the study of convergence rate of RPIM in case of boundary-value problem.

\subsection{RBF interpolation error}

The accuracy of RPIM shape functions used for surface fitting is determined. The fitting of functions is based on the nodal function values generated at regularly as well as irregularly distributed nodes. The procedure carried out in order to test the quality of the surface fitting is:

1. we define a set of nodes spread over the domain,

2. we define a set of $n$ evaluation points $\mathbf{x}_{i}$ (different from the previous set of nodes),

3. we compute at each point $\mathbf{x}_{i}$ the difference between the exact value $f_{i}$ of the surface function and its interpolated value $\tilde{f}_{i}$, 
4. we calculate an average error $e_{t}$, defined bellow, for all the points $\mathbf{x}_{i}$,

$$
e_{t}=\frac{1}{n} \sum_{i=1}^{n}\left|\frac{\tilde{f}_{i}-f_{i}}{f_{i}}\right|
$$

To construct the RPIM shape function the inverse of the matrix G (Eq. (3.9c)) is computed, the numerical inversion of matrix $\mathbf{G}$ affects the accuracy of interpolation [23, 24]. Therefore, a second indicator is added to the interpolation error which is the 2-norm condition number of matrix $\mathbf{G}$.

\subsubsection{Shape parameters analysis}

The shape parameters of RBF can have a significant impact on the accuracy and robustness of the interpolation, and because there is no rule governing the rational choice of these parameters, this was and stay very problematic when this kind of interpolation is used. A method for finding an optimal shape parameter is needed. Several works have been developed for that purpose. The paper of Franke [25] deal with the convergence study of the RBF interpolation, particularly the multiquadrique (MQ). Hardy [26] recommended the value $\alpha_{c}=0.815 d_{s}$ where $d_{s}$ is the influence domain. Rippa [27] proposed an optimization algorithm for the choice of the rational parameters, which was improved by Scheuerer [28]. Wang and Liu [29,30] studied the optimal values of RBF-MQ and EXP shape parameters in 2D and 3D [31], and they confirmed that the condition number of matrix $\mathrm{G}$ directly affects the accuracy of RBF interpolation. R. Li et al. [32] found a range of optimal values of the RBF-MQ shape parameters for the hybrid radial boundary node method. All the above works and others can be summarized in the following main classes [61] of strategies for searching optimal RBF shape parameters: the trial and error strategy, the power function, cross validation approach, contour-Pade algorithm.

In this work, we adopt the most used algorithm known as "leave-one-out" cross validation (LOOCV) [27] and compare the result of optimal shape parameter obtained by this algorithm with those deducted from the average error (Eq. (7.1)). The problem can be formulated as finding $c$, a parameter of the studied RBF, in order to minimize a "cost function" given by the norm of an error vector $E(c)$ with components

$$
E_{i}(c)=f_{i}-\sum_{j=1, j \neq i}^{N} a_{j}^{(i)} r\left(\left\|x_{j}-x_{i}\right\|, c\right)
$$

Here the sum in the above equation is the function value predicted at the $i$-th data point using RBF interpolation based on a set of nodes that exclude the same $i$-th node. The quality of the fit is determined by the norm of the vector of errors $E_{i}$. In [27] the author presented several examples where he uses the $l_{1}$ and $l_{2}$ norms. In our case, we will use the maximum norm. Note that we use the Matlab code "LOOCV2D" provided in [61].

RPIM shape functions are constructed in a domain of $(x, y) \in[-1,1] \times[-1,1]$ using three sets of $5 \times 5=25$ nodes patterns generated within this domain (Fig. 1). A set of 


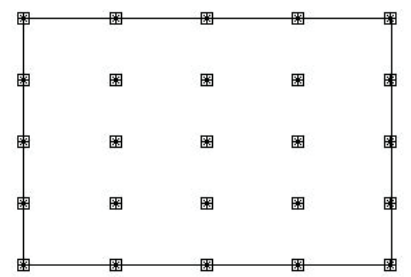

(a)

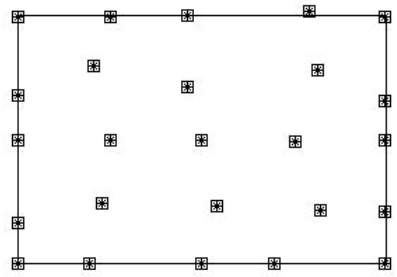

(b)

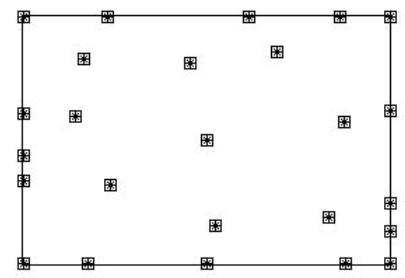

(c)

Figure 1: The three sets of nodes patterns.

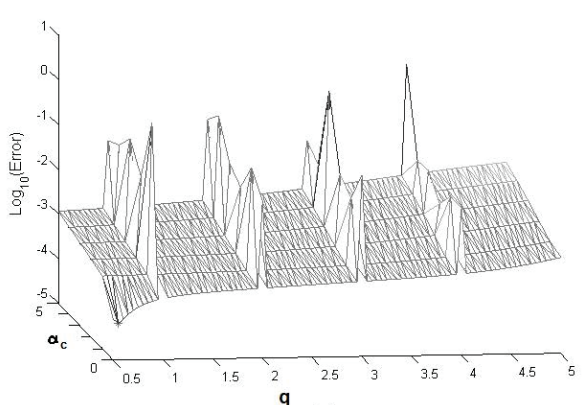

(a)

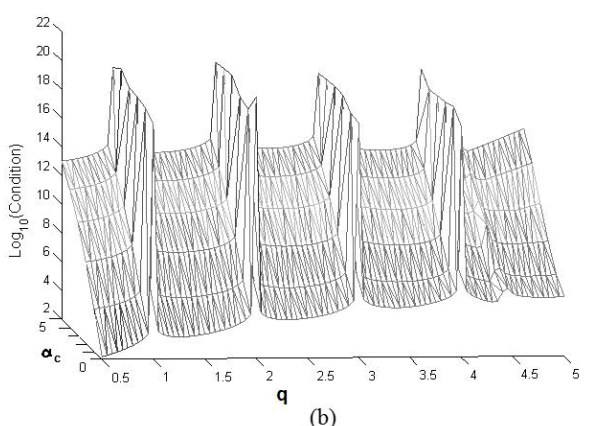

(b)

Figure 2: Effect of shape parameters $q$ and $\alpha_{c}$ of RBF-MQ on (a) interpolation error (b) condition number of matrix $\mathbf{G}$ for nodes pattern (a).

100 points are defined as evaluation points. The RBFs previously mentioned are used (Multiquadric (MQ), Gaussian (EXP), Piecewise polynomial (Pp)) (Table 1), in addition of an RBF with compact support (Wu-C2) (Eq. (2.2)). The linear polynomial basis is added to the RBFs. The evaluation procedure can be prescribed as follows: for a fixed number of nodes and sampling points, and for a range of RBF shape parameters, the interpolation error of curve fitting is calculated for function defined by:

$$
f(x, y)=\sin \left(\sqrt{\left(x^{2}+y^{2}\right)}\right) .
$$

For this example, a large influence domain is chosen that includes all nodes of the problem, in order to study only the effect of the RBF parameters, and once the good parameters are selected, it is possible to choose dimension of influence domain. Note that we assume that the parameter $c$ to be optimized by LOOCV algorithm is equal to $\alpha_{c}\left(c=\alpha_{c} d_{c}\right.$ and $\left.d_{c}=1\right)$, and the concerned RBFs are only MQ and EXP.

Different sets of nodes patterns (Fig. 1) are employed in order to evaluate the capacity of RPIM to deal with problems where nodes distribution are irregular. Through Figs. 2 to 9 , conclusions are given as follows:

1. Results of the optimal $\alpha_{c}$ obtained by LOOCV algorithm are listed bellow: we can 


\begin{tabular}{||c|c|c||}
\hline \multirow{2}{*}{ RBFs } & \multicolumn{2}{|c|}{ Optimal $\alpha_{c}$} \\
\cline { 2 - 3 } & Graphical & LOOCV \\
\hline MQ & 0.23 & 0.42 \\
\hline EXP & 8.35 & 6.97 \\
\hline
\end{tabular}

say that the results of LOOCV algorithm are in good agreement with those deducted from Figs. 2 and 3).

2. In order to find the good shape parameters of RBF, it would be necessary to find the just balance between the error of interpolation and the condition number of matrix $\mathbf{G}$, and that is the case with the LOOCV [27].

3. Singular values found on the two basis $M Q$ and $P p$ are resulting from the fact that these radial basis functions are not defined as conditionally positive definite functions at this particular values. This is the reason why the condition number of matrix $\mathbf{G}$ is important ( $\mathbf{G}$ is singular), this situation involve that the linear system 3.9c becomes ill-posed.

4. Both Gaussian (EXP) and Wu-C2 basic functions present no singular behavior for all shape parameters values.

5. One notice through Figs. 6 to 9, that the way nodes are distributed has no significant effect on the quality of RBF interpolation.

\subsubsection{Dimensions of the influence domain}

The effect of influence domain size (with circular shape) on the accuracy of RBF interpolation is studied. The domain is discretized by 529 nodes distributed in an irregular manner. By increasing gradually the size of the influence domain, the number of nodes inside this domain will increase, then we proceed to the calculation of interpolation error and condition number of matrix G. The RBFs shape parameters are simply selected

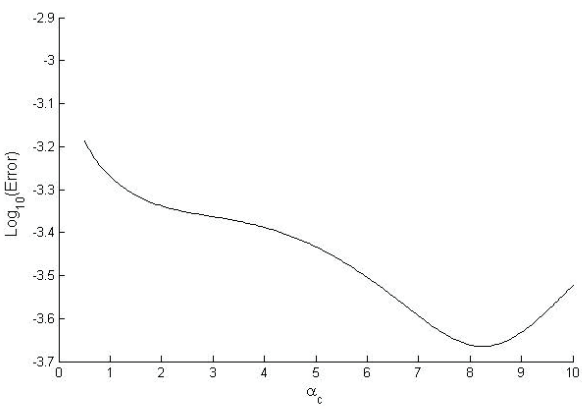

(a)

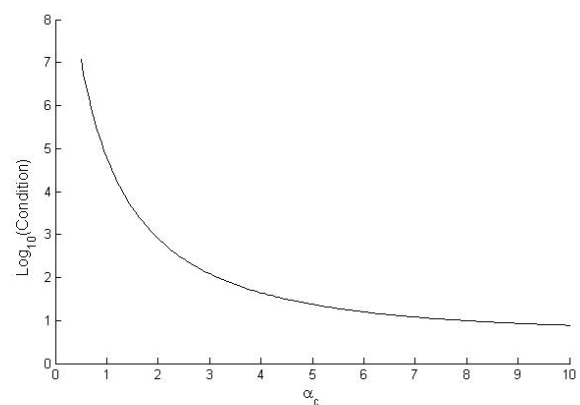

(b)

Figure 3: Effect of shape parameter $\alpha_{c}$ of RBF-EXP on (a) interpolation error (b) condition number of matrix $\mathbf{G}$ for nodes pattern (a). 


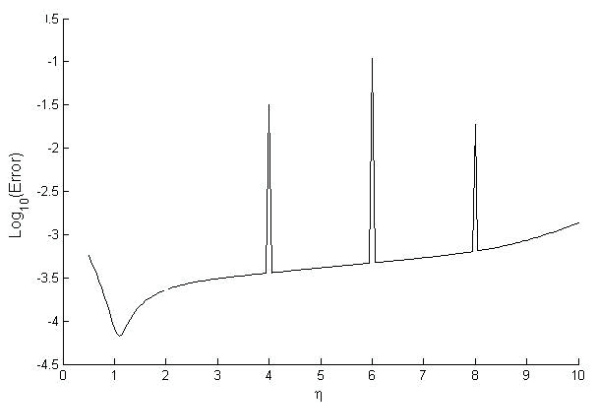

(a)

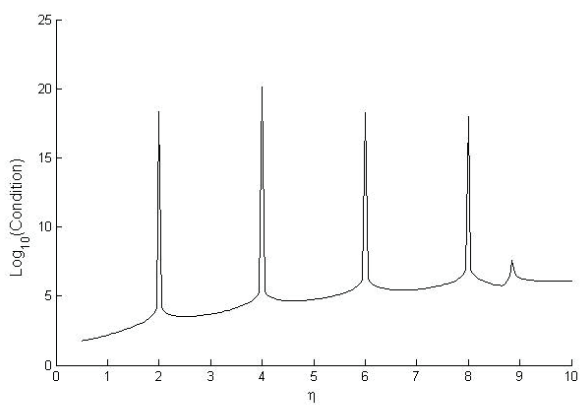

(b)

Figure 4: Effect of shape parameter $\eta$ of RBF-Pp on (a) interpolation error (b) condition number of matrix G for nodes pattern (a).

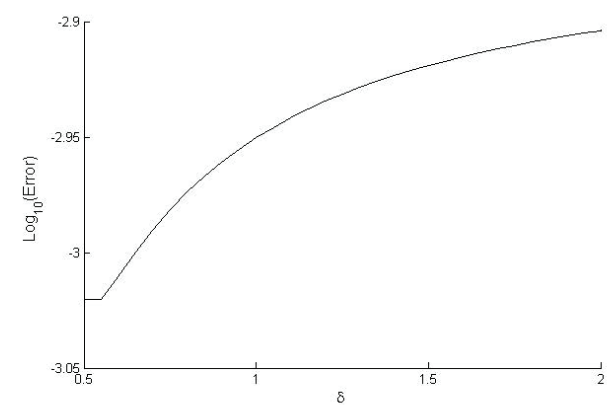

(a)

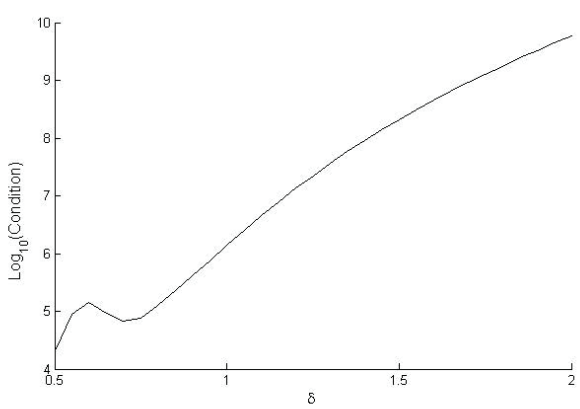

(b)

Figure 5: Effect of shape parameter $\delta$ of RBF-Wu-C2 on (a) interpolation error (b) condition number of matrix $\mathbf{G}$ for nodes pattern $(a)$.

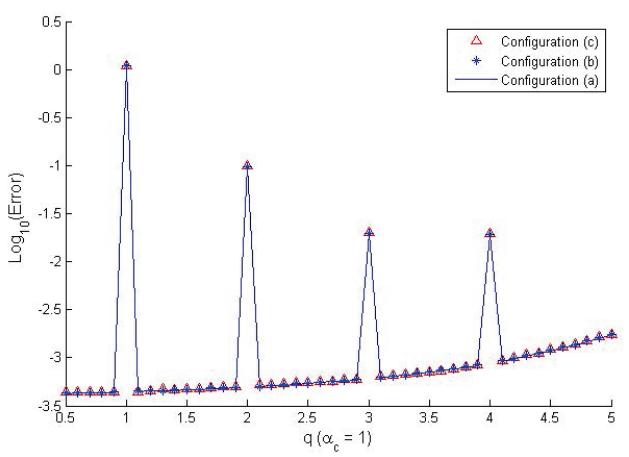

Figure 6: Effect of shape parameter $q$ of RBF-MQ on interpolation error with different sets of nodes patterns.

such that singular values are avoided: $\mathrm{MQ}\left(q=1.3, \alpha_{c}=0.5\right), \operatorname{EXP}\left(\alpha_{c}=4\right), \operatorname{Pp}(\eta=3)$, $\mathrm{Wu}-\mathrm{C} 2\left(\delta_{c}=\right.$ influence domain). The obtained results are illustrated in Figs. 10, 11 and the 


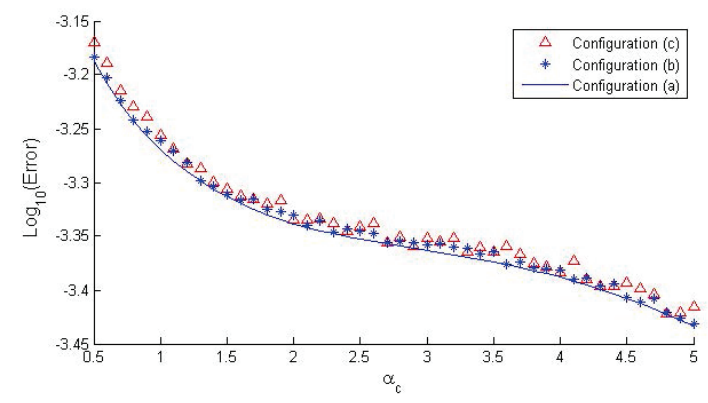

Figure 7: Effect of shape parameter $\alpha_{c}$ of RBF-EXP on interpolation error with different sets of nodes patterns.

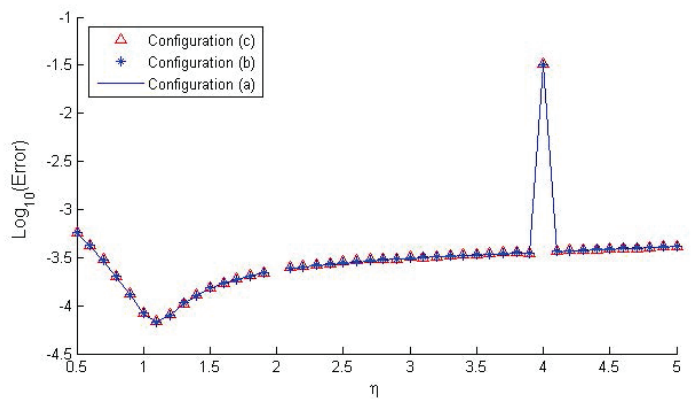

Figure 8: Effect of shape parameter $\eta$ of RBF-Pp on interpolation error with different sets of nodes patterns.

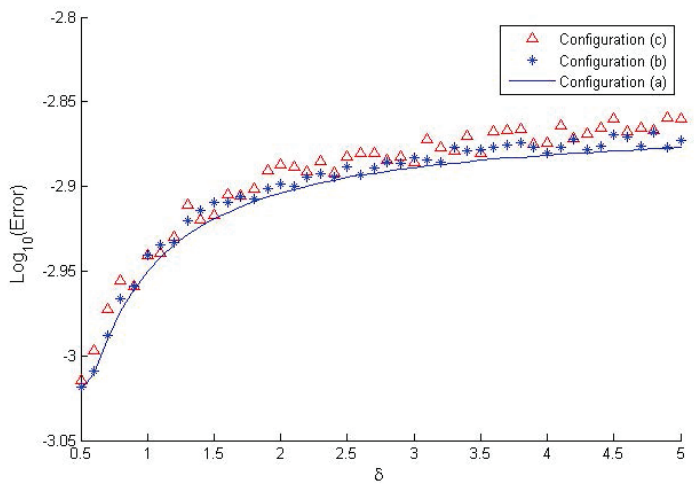

Figure 9: Effect of shape parameter $\delta$ of RBF-Wu-C2 on interpolation error with different sets of nodes patterns.

following conclusions are drawn:

1. The condition number of matrix $\mathrm{G}$ increase systematically with the dimensions of the influence domain, thus more the number of nodes inside this domain increases more the matrix $\mathbf{G}$ is badly conditioned. 


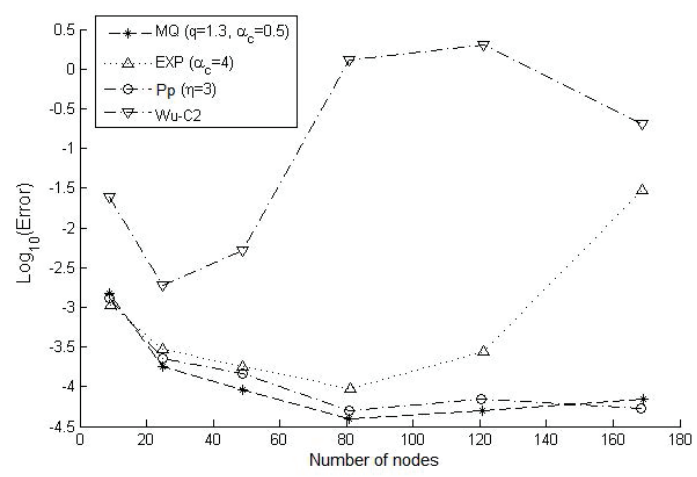

Figure 10: Effect of influence domain dimension on interpolation error with different types of RBF.

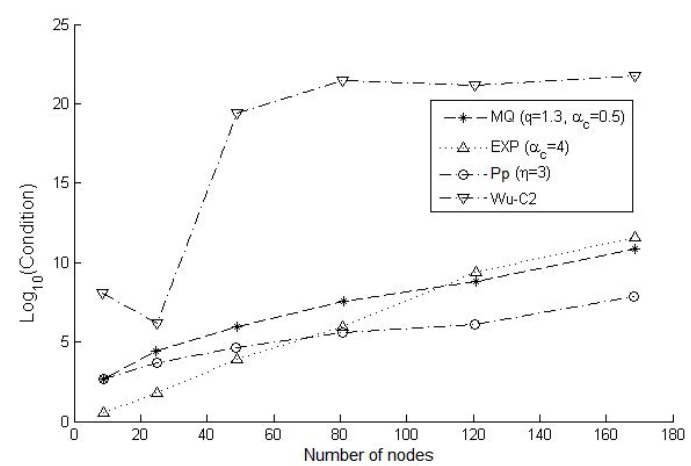

Figure 11: Effect of influence domain dimensions on condition number of matrix $\mathbf{G}$ by different types of RBF.

2. The interpolation error does not decrease systematically by increasing the number of nodes inside the influence domain, because, firstly the more the number of nodes in the interpolation is important more the number of computational arithmetic operations is important, this introduce calculation errors, secondly more the number of nodes is important more the condition number of matrix $\mathbf{G}$ is larger this means it is ill-conditioned and its inversion will cause larger error.

3. Larger influence domain means higher number of nodes included in interpolation, and implies increase of floating points operations (Fig. 12).

4. From this analysis it is recommended to use 6 to 25 nodes in the influence domain that generates good results. A bigger or smaller number of nodes would lead to larger numerical error.

5. The technique based on the concept of natural neighbor $[2,33,34]$ is in good accordance with this choice. 


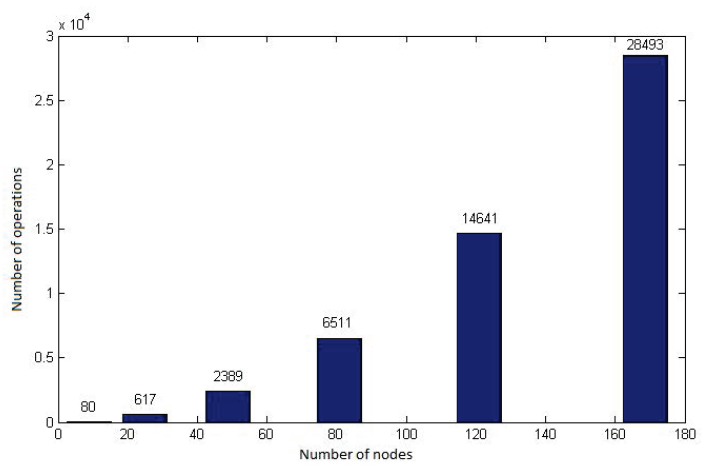

Figure 12: Effect of influence domain dimensions on the number of arithmetic operations.

\subsection{Numerical experiments of 2-D solid mechanics problems}

\subsubsection{Error index}

We now propose to study the behavior of RBFs in solving 2D mechanical problems. To do that the RBFs are used in the associated Galerkin weak form. In order to evaluate the accuracy of the obtained solutions, the definition of specific errors is necessary. The relative error of displacements is defined as follows:

$$
e_{d}=\frac{\|u-\widehat{u}\|_{L^{2}}}{\|u\|_{L^{2}}}=\frac{\left(\int_{\Omega}(u-\widehat{u})^{T}(u-\widehat{u}) d \Omega\right)^{1 / 2}}{\left(\int_{\Omega}(u)^{T}(u) d \Omega\right)^{1 / 2}},
$$

where $\hat{u}$ and $u$ are the displacements computed by the RPIM and the exact analytical solution, respectively. The error of energy is defined as follows:

$$
e_{E d e f}=\frac{\left(\int_{\Omega} \frac{1}{2}(\varepsilon-\widehat{\varepsilon})^{T} c(\varepsilon-\widehat{\varepsilon}) d \Omega\right)^{1 / 2}}{\left(\int_{\Omega} \frac{1}{2}(\varepsilon)^{T} c(\varepsilon) d \Omega\right)^{1 / 2}},
$$

where $\widehat{\varepsilon}$ and $\varepsilon$ are strain tensors obtained from the RPIM and the exact analytical closedform solution, respectively. In order to evaluate the convergence rates of RPIM methods, we introduce a characteristic length " $h$ ". For a grid of triangular T3 background cells:

$$
h=\sqrt{\frac{2 A_{\Omega}}{n_{c}}},
$$

and for a grid of quadrilateral Q4 background cells:

$$
h=\sqrt{\frac{A_{\Omega}}{n_{c}}}
$$

where $A_{\Omega}$ is the area of the problem domain and $n_{c}$ the number of integration cells. 


\subsubsection{Cantilever beam problem}

This problem is widely used to select the optimal parameters of RPIM [29-32] and almost all reported conclusions are obtained from this benchmarking numerical experiment. Consider a cantilever beam shown in Fig. 13. The beam is fixed at the left end and subjected to a parabolic traction force at the right end, $P$ being the resultant of this parabolic traction. The analytical solution is defined as follows [54]:

$$
\begin{aligned}
& u(x, y)=-\frac{P y}{6 E I}\left[(6 L-3 x) x+(2+v)\left(y^{2}-\frac{D^{2}}{4}\right)\right], \\
& v(x, y)=\frac{P}{6 E I}\left[3 v y^{2}(L-x)+(4+5 v) \frac{D^{2} x}{4}+(3 L-x) x^{2}\right],
\end{aligned}
$$

where the moment of inertia $I$ of the beam is given by $I=D^{3} / 12$. The beam parameters are taken as $P=-1000 \mathrm{~N}$, the Young modulus $E=3 \times 10^{7} \mathrm{MPa}$, the poisson coefficient $v=0.3, D=12 \mathrm{~mm}, L=48 \mathrm{~mm}$. The corresponding stresses are:

$$
\begin{aligned}
& \sigma_{x x}(x, y)=-\frac{P(L-x) y}{I}, \quad \sigma_{y y}=0, \\
& \tau_{x y}(x, y)=\frac{P}{2 I}\left[\frac{D^{2}}{4}-y^{2}\right] .
\end{aligned}
$$

\section{Shape parameters analysis}

For this test, two configurations of nodes will be used: 325 nodes distributed in a regular and irregular way respectively (Fig. 14). Only two radial basis functions are handled: the multiquadric (MQ) and the Gaussian (EXP). Here circular influence domain circumvents 8 to16 nodes is used for each Gaussian point. Linear polynomial terms are included for the RBF interpolation. For the integration $4 \times 4=16$ Gauss points are used for each cell of a set of $288(24 \times 12)$ background cells (Fig. 15). First, the effect of the multiquadric shape parameters (MQ) is studied, results are illustrated in Figs. 16 and 17.

1. For a uniform distribution of nodes, the error is minimal for $q$ between $1.5-2$ and all mentioned $\alpha_{c}(0.5,1,1.5,2)$. We avoided the value of $q=2$ because it is the particular value which makes the matrix $\mathbf{G}$ singular or strongly ill-conditioned.

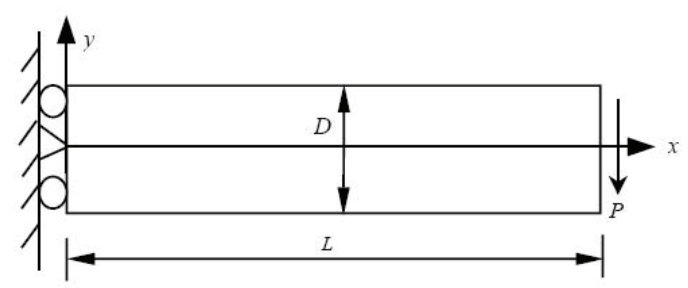

Figure 13: Cantilever beam problem. 


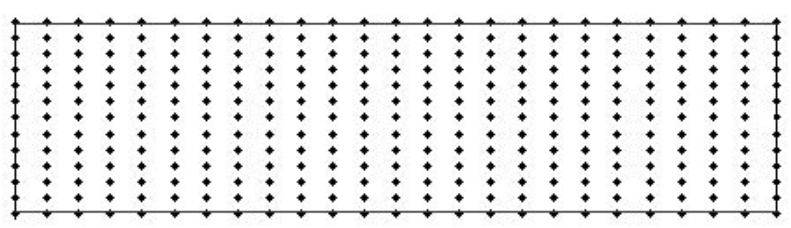

regular nodes configuration

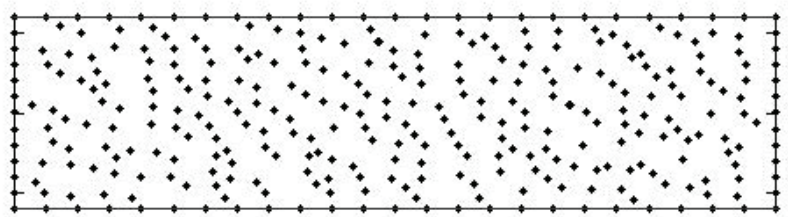

irregular nodes configuration

Figure 14: Nodes configurations of the cantilever beam problem.

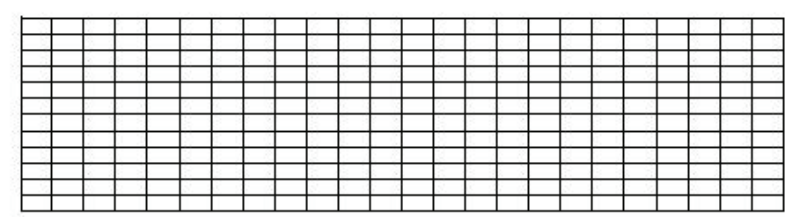

Figure 15: $(24 \times 12)$ background cells for numerical integration.

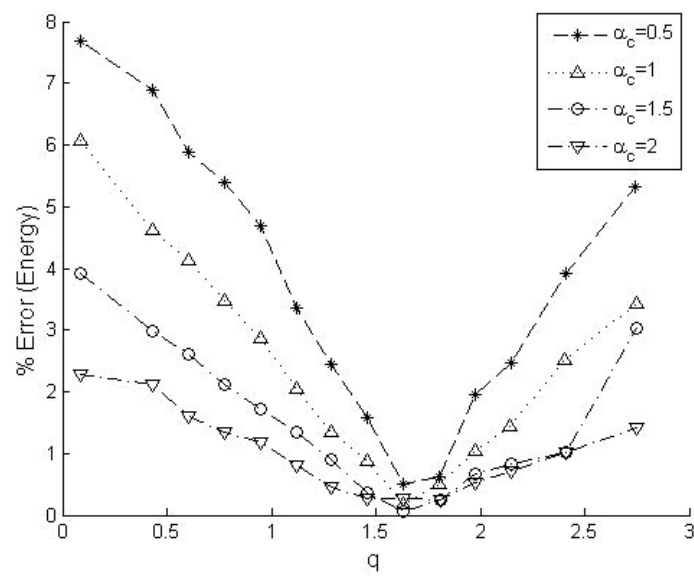

Figure 16: Effect of RBF-MQ shape parameters on energy error for regular nodes distribution. 


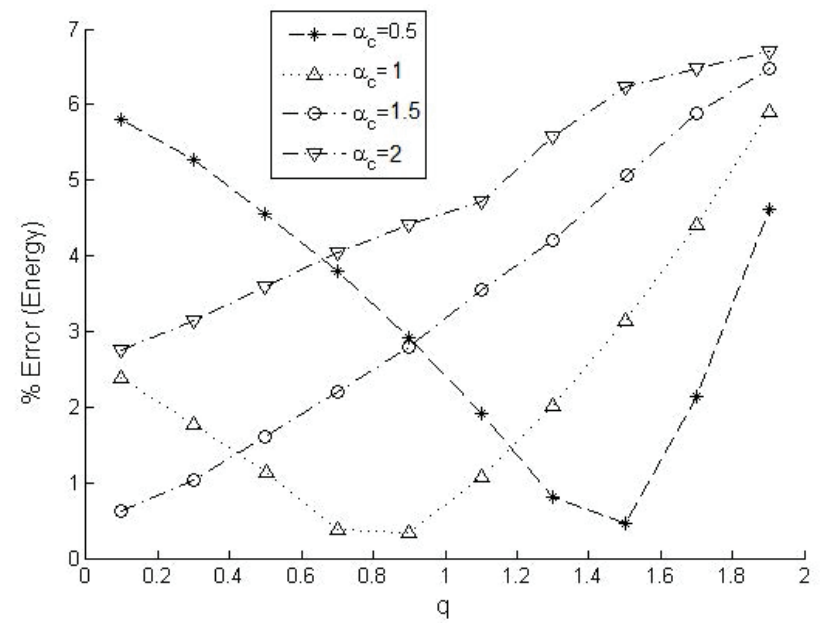

Figure 17: Effect of RBF-MQ shape parameters on energy error for irregular nodes distribution.

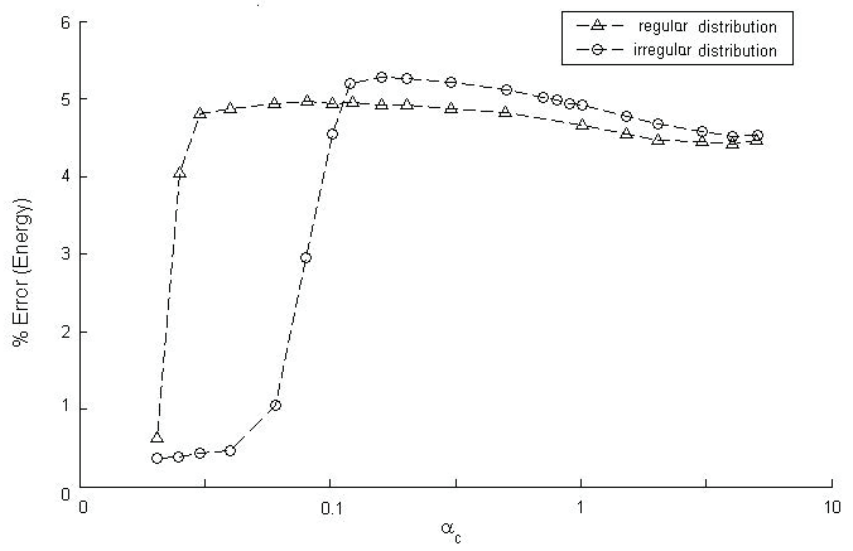

Figure 18: Effect of RBF-EXP shape parameter on energy error for regular/irregular nodes distribution.

2. For an irregular nodes distribution, the optimal value of $q$ varies according to the value of $\alpha_{c}$.

3. The recommended values for the Multiquadric (MQ) are: $\alpha_{c}=0.5 \sim 2$ and $q=0.4 \sim 2.2$ with the exception of the singular values (integers).

Secondly, the effect of the Gaussian shape parameter (EXP) is illustrated in Fig. 18.

1. For a uniform node distribution, the values of energy error remain relatively invariant for $\alpha_{c}>0.03$. 
2. For irregular nodes distribution, the behavior of the error according to $\alpha_{c}$ is almost the same that for uniform configuration.

3. The recommended values for Gaussian (EXP) are: $\alpha_{c}=0.2 \sim 1$.

\section{Dimensions of the influence domain}

In this analysis, a configuration of 975 (39x25) nodes uniformly distributed is adopted. The same configuration is used for quadrilateral background cells (Fig. 19). Four configurations of shape parameters for the RBF are used: RBF-MQ with $q=1.3$ and $\alpha_{c}=0.5$ and $q=1.9$ and $\alpha_{c}=1.5$, RBF-EXP with $\alpha_{c}=0.2$ and $\alpha_{c}=0.6$. The number of Gauss points is maintained to 16 points by cell.

1. Large size of the influence domain does not imply systematically a good precision, in fact quite the opposite. Moreover the number of arithmetic operations required for the calculation increase, which would have a consequence on increasing the CPU time (Fig. 21).

2. The number of nodes recommended to reach a good quality is of $9 \sim 30$ nodes inside the influence domain $(d c=2.5$ in our case).

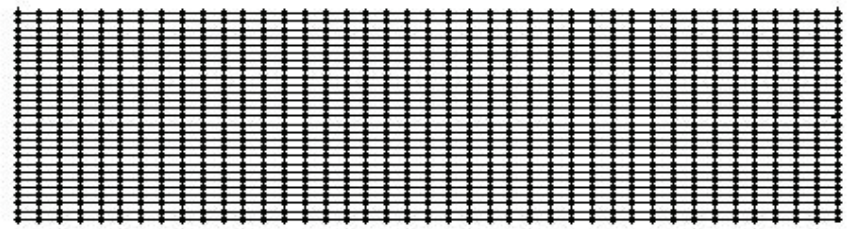

Figure 19: The two combined configurations of $975(39 \times 25)$ nodes and $1040(40 \times 26)$ background cells.

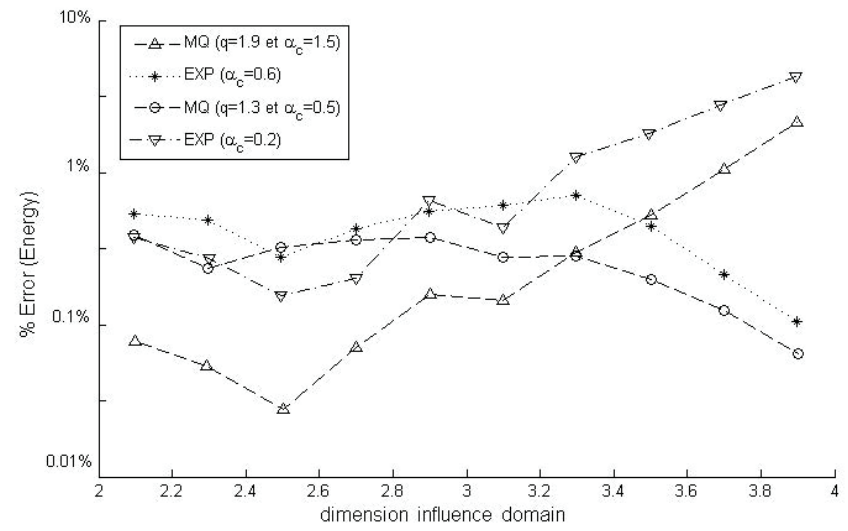

Figure 20: Effect of influence domain dimensions on energy error. 


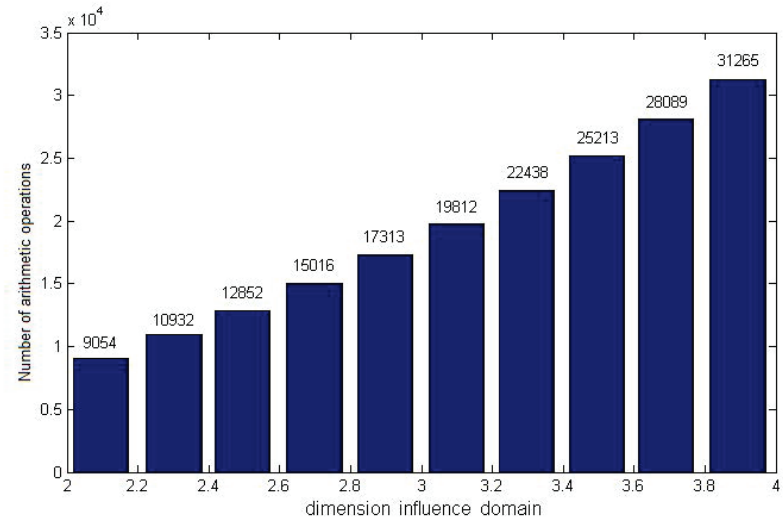

Figure 21: Effect of influence domain dimensions on the number of computational arithmetic operations.

\section{Number of Gauss points}

To analyze how the number of Gauss points by a cell used in Gauss integration technique affects the accuracy of RPIM, certain parameters have to be fixed: for nodal discretization and background cells the previous uniform configurations are used (Figs. 14 and 15). Two radial basis functions are considered: the RBF-MQ with $q=1.9$ and $\alpha_{c}=1.5$, the RBF-EXP with $\alpha_{c}=0.2$, the influence domain is being determined by 6 to 12 nodes inside. Fig. 22 shows the variation of the energy error according to the number of Gauss points by cell. It is noticed that more the number of gauss points will be important, better is the quality of RPIM results until a relative stabilization for a number larger than $64=8 \times 8$ quadrature points. On the other hand, it would be necessary to pay attention to the CPU time for numerical calculations which tends to increase proportionally with the

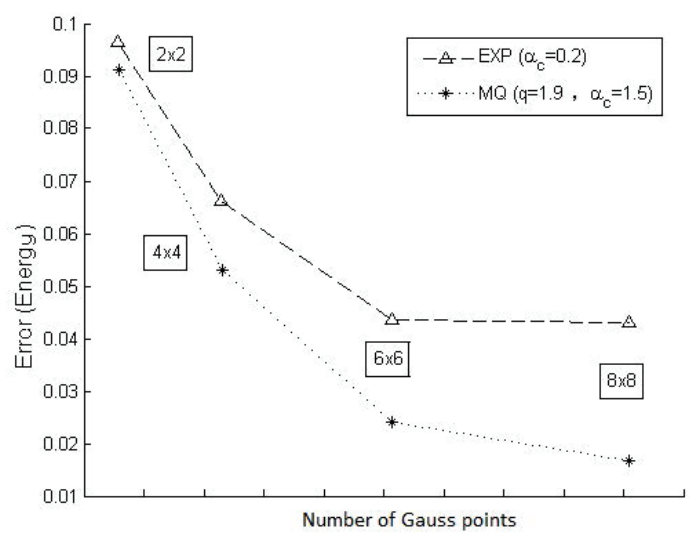

Figure 22: Energy error function of the number of Gauss points. 
increase of used Gauss points. It is recommended to take a $4 \times 4$ up to $6 \times 6$ Gauss points for rectangular cells.

\section{Effect of the integration technique}

The objective of this part is to compare two numerical integration techniques: the classical Gauss integration (the RPIM) and the Stabilized conforming nodal integration (SCNI, the SRPIM). The chosen radial basic function is the Gaussian (RBF-EXP) with $\alpha_{c}=0.2$, with addition of 1 st order polynomial terms. The influence domain being determined with 8-16 nodes inside. A total of $6 \times 6$ Gauss points are used for the classic Gauss quadrature, and 8 points (four cell vertices and four mid-points on edges) for the SCNI.

Five set of regularly distributed nodes/integration quadratic cells pattern are used for this example: 1150, 798, 520, 360, 284.

First, it is noticed (Figs. 23 and 24) that the convergence rates are quite close for all the approaches. However, the error level of meshfree methods (RPIM, SRPIM) are better than

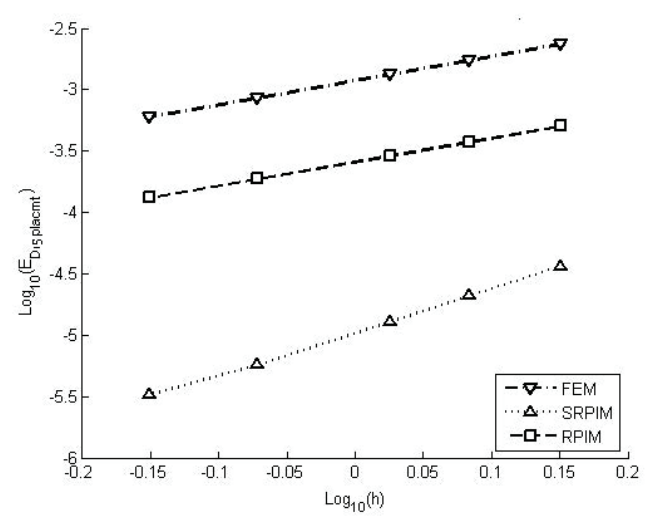

Figure 23: Relative error in displacement calculated for FEM, RPIM, SRPIM.

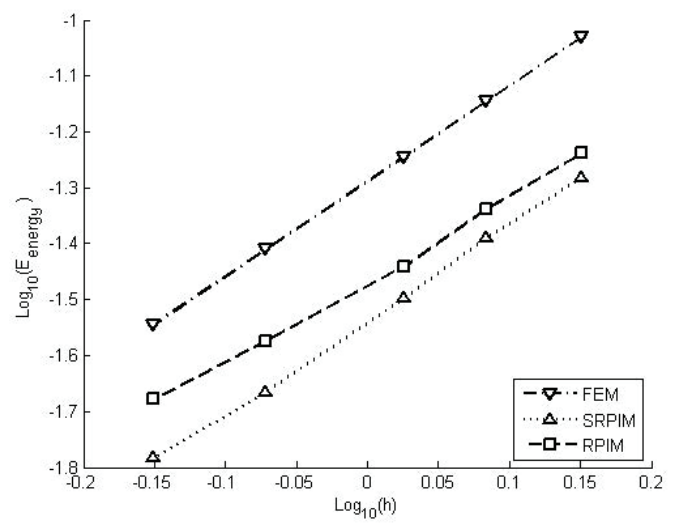

Figure 24: Relative energy error calculated for FEM, RPIM and SRPIM. 
those obtained by the FEM, due to the higher order of RBF interpolation comparing with interpolation used in FEM linear triangular element. Secondly, among RPIM meshfree methods the one who presents a smaller error is SRPIM.

\subsection{Infinite plate with a circular hole}

A plate with a central circular hole with radius $a=1 \mathrm{~m}$ subjected to a unidirectional tensile force of $1 \mathrm{~N} / \mathrm{m}$ in $x$-direction is studied. Due to symmetry, only the upper right quadrant of the plate is modeled as shown in Fig. 25. Symmetry conditions are imposed on the left and bottom edges. The inner boundary (the hole) is traction free. Plane strain conditions are assumed, and the problem parameters are: $L=5 \mathrm{~m}, a=1 \mathrm{~m}, E=10^{3} \mathrm{~Pa}, v=0.3, P=1 \mathrm{~N} / \mathrm{m}$. The analytical solution for an infinite plate [54], is:

$$
\left\{\begin{array}{l}
u_{x}=\frac{a}{8 \mu}\left[\frac{r}{a}(\kappa+1) \cos \theta+2 \frac{a}{r}((1+\kappa) \cos \theta+\cos 3 \theta)-2 \frac{a^{3}}{r^{3}} \cos 3 \theta\right], \\
u_{y}=\frac{a}{8 \mu}\left[\frac{r}{a}(\kappa-3) \sin \theta+2 \frac{a}{r}((1-\kappa) \sin \theta+\sin 3 \theta)-2 \frac{a^{3}}{r^{3}} \sin 3 \theta\right]
\end{array}\right.
$$

where $(r, \theta)$ are the polar coordinates with $\theta$ measured counter-clockwise from the positive $x$ axis, $\mu=E /(2(1+v))$ at $\kappa=3-4 v$. The analytical solution for the stresses of an

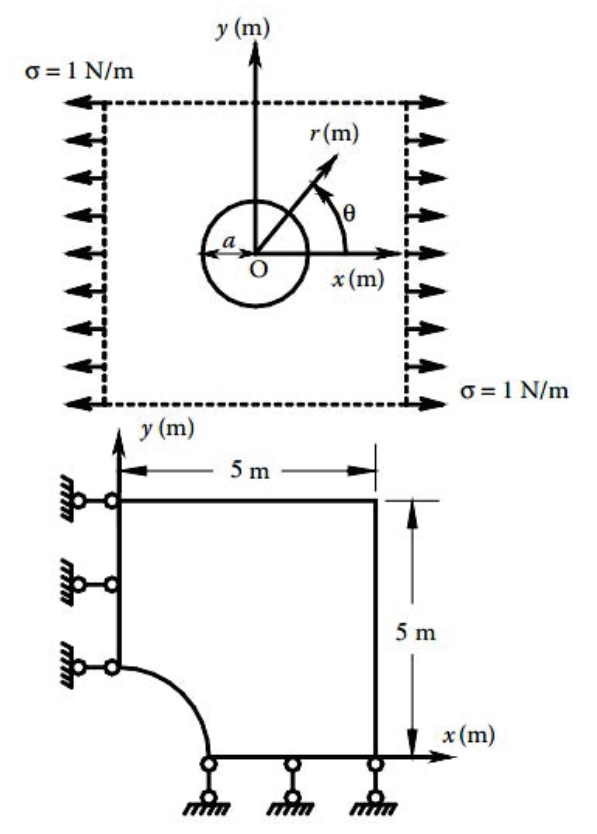

Figure 25: Geometry of the infinite plate with a hole problem. 


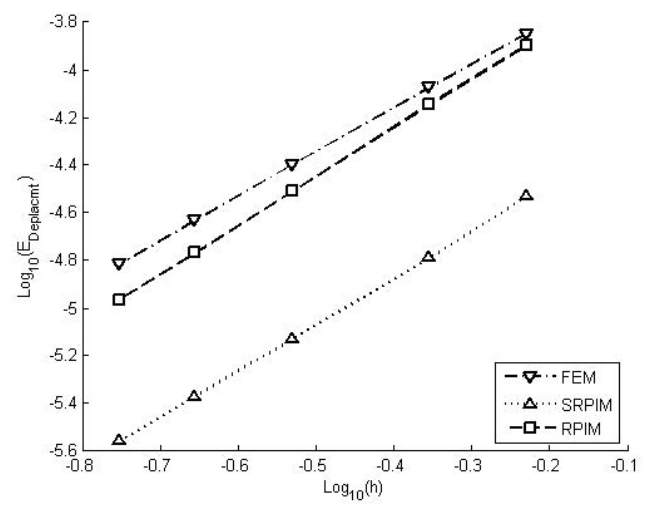

Figure 26: Relative error in displacement calculated for FEM, RPIM, SRPIM.

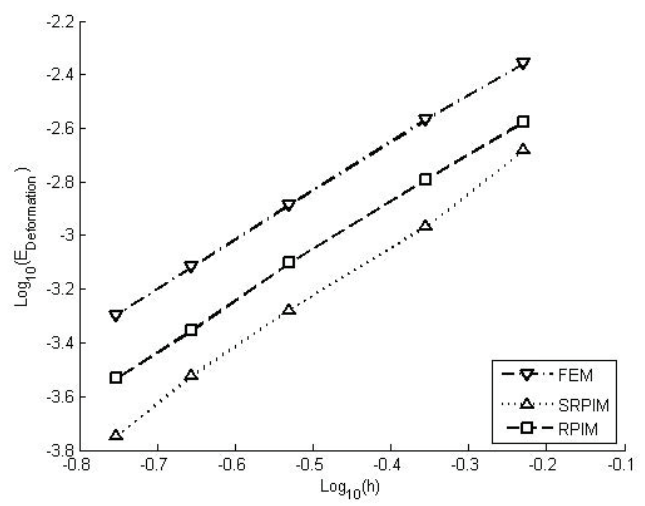

Figure 27: Relative energy error calculated for FEM, RPIM and SRPIM.

infinite plate is:

$$
\begin{aligned}
& \sigma_{x}(x, y)=1-\frac{a^{2}}{r^{2}}\left\{\frac{3}{2} \cos 2 \theta+\cos 4 \theta\right\}+\frac{3 a^{4}}{2 r^{4}} \cos 4 \theta, \\
& \sigma_{y}(x, y)=-\frac{a^{2}}{r^{2}}\left\{\frac{1}{2} \cos 2 \theta-\cos 4 \theta\right\}-\frac{3 a^{4}}{2 r^{4}} \cos 4 \theta, \\
& \sigma_{x y}(x, y)=-\frac{a^{2}}{r^{2}}\left\{\frac{1}{2} \sin 2 \theta-\sin 4 \theta\right\}+\frac{3 a^{4}}{2 r^{4}} \sin 4 \theta .
\end{aligned}
$$

The displacement boundary conditions (symmetry) are given by: on the edge of $x=u_{y}=0$, on the edge of $y=u_{x}=0$. On the edges $x=5$ and $y=5$ we impose the displacement given by the analytical solution (Eq. (7.10)).

As in the previous example, five nodes configurations are used for this problem. The total number of nodes used is: 169, 289, 625, on 1089 and 1681. Figs. 26 and 27, illustrate convergence rates obtained from RPIM, SRPIM and FEM. It is noticed that the level of 
error obtained with meshless methods are better than those obtained with the FEM, and among the RPIM methods, SRPIM presents a better precision in terms of displacement error and energy error. This confirms the quality of the SCNI integration.

\subsection{Distortion analysis}

The example treated in this test is close to the previous cantilever beam test (Fig. 13), but with a different geometry and load (Fig. 28). This geometry is discretized by various nodes configurations (Fig. 29). The chosen Radial basic function is the Gaussian (EXP) with $\alpha_{c}=0.2$. The influence domain is defined by 8-16 nodes inside for the RPIM, and 8 points (four cell vertices and four mid-points on edges) for the SRPIM. A total of $6 \times 6$ Gauss points are used for the classic Gauss quadrature.

The exact solution of the stress field [62] is:

$$
\sigma_{x x}(x)=\frac{3}{2} y, \quad \sigma_{y y}(x)=0, \quad \sigma_{x y}(x)=0 .
$$

The strain field is given by:

$$
\begin{aligned}
& \varepsilon_{x x}(x)=\frac{3 E(1-v)}{2(1-v)(1-2 v)} y, \\
& \varepsilon_{y y}(x)=\frac{3 E v}{2(1-v)(1-2 v)} y, \varepsilon_{x y}(x)=0 .
\end{aligned}
$$

As noticed in Figs. 30 and 31, for the first regular nodes distributed configuration (configuration (1)), all RPIM methods give satisfactory results and better than those obtained by FEM (with linear triangular element mesh). But it is noticed that the error increases with the distortion of the nodes distribution, and this in spite of the fact that the influence domain guarantees a minimum number of nodes. The method which seems the least affected by this distortion is the SRPIM (with SCNI technique).

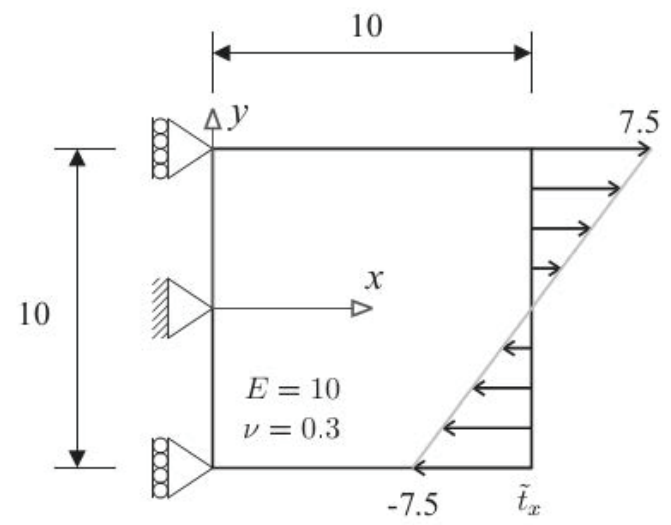

Figure 28: Model used to examine distortional effects. 

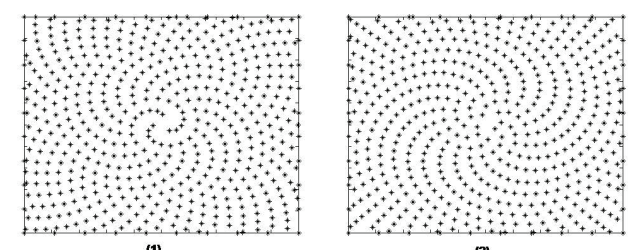

(2)

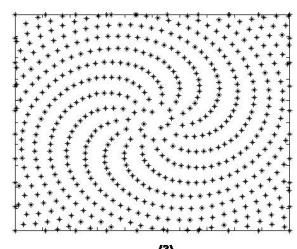

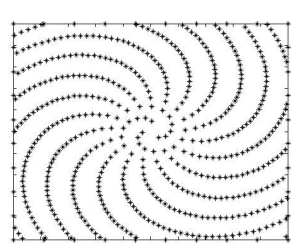

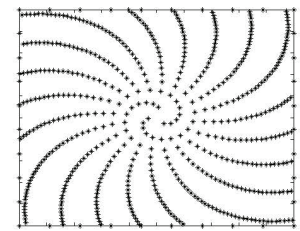

Figure 29: Grids used to examine the influence of distortion.

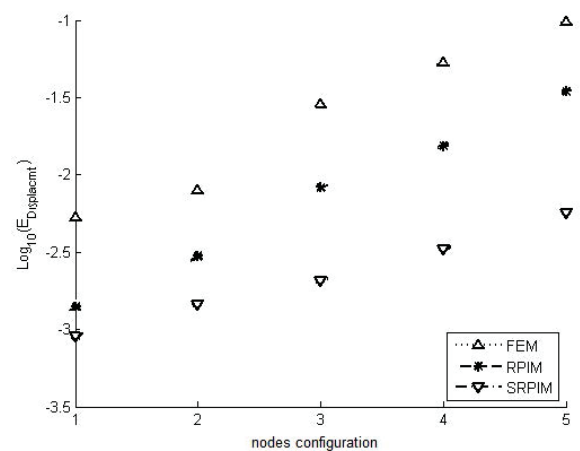

Figure 30: Relative error of displacement on results obtained by FEM, RPIM and SRPIM with several nodes configurations.

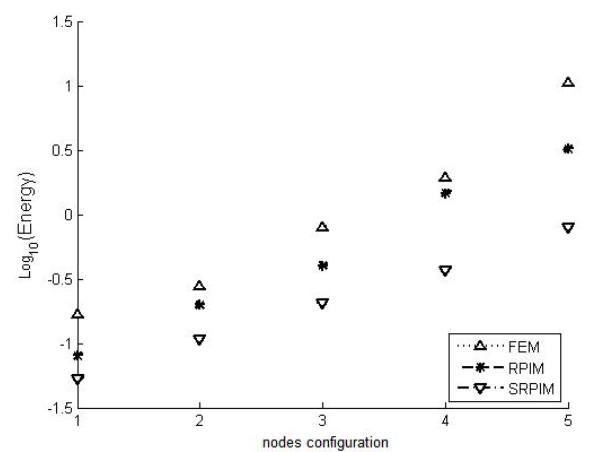

Figure 31: Energy error on results obtained by FEM, RPIM and SRPIM with several nodes configurations. 


\section{Conclusions}

A numerical analysis was performed on the RPIM meshless methods with two integration schemes. The accuracy of RBF shape functions and linear elasticity resolution was investigated. It has shown that the Radial basis interpolation has a positive effect on the global accuracy of the RPIM. Shape parameters have important effects on the condition number of the matrix $\mathbf{G}$, this condition number heavily affects the accuracy of interpolation. Thus, the range of good shape parameters should balance the accuracy and the condition number. Through numerical experiments, a range of shape parameters was found which give good results. For regular nodal distribution, when the number of nodes inside the influence domain ranges from 6 to 25, the accuracy is high. When adding linear polynomial term and an appropriate influence domain size, the shape parameters can be chosen from a larger range of values avoiding singular ones. Nodal distribution had little effect on the accuracy of RPIM interpolation. Furthermore it is observed that the strainsmoothing integration scheme is less sensitive to distortion than the Gaussian integration scheme and gives excellent results compared to a classical Gaussian integration.

The main contributions of this work can be summarized as follows: a detailed comparative study was made between two versions of RBF based meshless methods: the RPIM and the S-RPIM. The first classical method (RPIM) is widely used in computational mechanics and was able to confirm its place as one of the major meshless methods. The second method, more recent than the first one, has a number of advantages with respect to the classical meshless methods. The examination of these advantages in terms of accuracy and flexibility was more than necessary, especially when a few works have been dedicated to draw attention to these properties. We mean by flexibility the capacity of giving immunity in situations of extreme mesh distortion where the Jacobian geometric mapping goes negative in the classical methods. This point was further reinforced in this paper, where we have shown the dual mesh distortion immunity of the RBF shape function in one hand and the strain-smoothing integration technique in the other hand, the combination of the two techniques result to a robust meshless method.

\section{References}

[1] N. S. LEE AND K. J. BATHE, Effects of element distortions on the performance of isoparametric elements, Int. J. Numer. Meth. Eng., 36 (1993), pp. 3553-3576.

[2] E. Cueto, N. Sukumar, B. Calvo, M. A. Martínez, J. Cegon̈ino and M. Doblarë, Overview and recent advances in natural neighbour galerkin methods, Archives of Computational Methods in Engineering, 10(4) (2003), pp. 307-384.

[3] J. SLATER, Electronic energy bands in metals, Phys. Rev., 45 (1934), pp. 794-801.

[4] R. FrAZER, W. JONES AND S. SKAN, Approximations to functions and to the solutions of differential equations, Great Britain Aero Counc. London. Rep. Memo., 1799(1) (1937), pp. 517-549.

[5] C. LANCZOS, Trigonometric interpolation of empirical and analytical functions, J. Math. Phys., 17 (1938), pp. 123-199. 
[6] M. B. LIU AND G. R. LIU, Smoothed Particle Hydrodynamics (SPH): an overview and recent developments, Archives of Computational Methods in Engineering, 17(1) (2010), pp. 25-76.

[7] L. LUCY, A numerical approach to testing the fission hypothesis, Astron. J., 82 (1977), pp. 10131024.

[8] B. Nayroles, G. Touzot And P. Villon, Generalizing the finite element method: diffuse approximation and diffuse elements, Comput. Mech., 10(5) (1992), pp. 307-318.

[9] W. K. LIU, S. JUN AND Y. F. ZHANG, Reproducing kernel particle methods, Int. J. Numer. Methods Eng., 20(8-9) (1995), pp. 1081-1106.

[10] W. K. LiU, Y. Chen, S. Jun, J. S. Chen, T. Belytschko, C. PAN, R. A. Uras and C. T. CHANG, Overview and applications of the reproducing Kernel Particle methods, Archives of Computational Methods in Engineering, 3(1) (1996), pp. pp. 3-80.

[11] Y. LU, T. BELYTSCHKO AND L. GU, A new implementation of the element free galerkin method, Comput. Methods Appl. Mech. Eng., 113(3-4) (1994), pp. 397-414.

[12] J. G. WANG AND G. R. LiU, Radial point interpolation method for elastoplastic problems, Proc. of the 1st Int. Conf. On Structural Stability and Dynamics, (2000), pp. 703-708.

[13] S. N. Atluri AND T. ZHU, A new Meshless Local Petrov-Galerkin (MLPG) approach in computational mechanics, Comput. Mech., 22(2) (1998), pp. 117-127.

[14] T. BELYTSCHKO, Y. LU AND L. GU, Element-free galerkin methods, Int. J. Numer. Methods Eng., 37(2) (1994), pp. 229-256.

[15] J. DOlBOW AND T. BELYTSCHKO, An introduction to programming the meshless Element Free Galerkin method, Archives of Computational Methods in Engineering, 5(3) (1998), pp. 207241.

[16] G. LiU AND Y. GU, A point interpolation method, The 4th Asia-Pacific Conference on Computational Mechanics, Singapore, (1999), pp. 1009-1014.

[17] G. R. LIU AND Y. T. GU, A point interpolation method for two-dimensional solids, Int. J. Num. Meth. Eng., 50 (2001), pp. 937-951.

[18] G. LIU AND Y. GU, A matrix triangularization algorithm for the polynomial point interpolation method, Comput. Methods Appl. Mech. Eng., 192(19) (2003), pp. 2269-2295.

[19] S. BEISSEL AND T. BELYTSCHKO, Nodal integration of the element-free galerkin method, Comput. Methods Appl. Mech. Eng., 139 (1996), pp. 49-74.

[20] J. CHEN, C. WU, S. YOON AND Y. YOU, A stabilized conforming nodal integration for galerkin mesh free methods, Int. J. Num. Meth. Eng., 50 (2001), pp. 435-466.

[21] J. CHEN, S. YOON AND C. WU, Nonlinear version of stabilized conforming nodal integration for galerkin meshfree methods, Int. J. Num. Meth. Eng., 53 (1993), pp. 2587-2615.

[22] M. GolberG, C. CHEN AND H. BOWMAN, Some recent results and proposals for the use of radial basis functions in the bem, Eng. Anal. Boundary Elements, 23 (1999), pp. 285-296.

[23] J. BOYD AND K. GILDERSLEEVE, Numerical experiments on the condition number of the interpolation matrices for radial basis functions, Appl. Numer. Math., 61(4) (2011), pp. 443-459.

[24] J. CHEN, W. Hu AND H. HU, Localized radial basis function with partition of unity properties, Progress on Meshless Methods Computational Methods in Applied Sciences, 11 (2009), pp. 37-56.

[25] R. FRANKE, Scattered data interpolation: tests of some methods, Math. Comput., 38(157) (1982), pp. 200-292.

[26] R. HARDY, Multiquadric equations of topography and other irregular surfaces, J. Geophys. Res., 176 (1971), pp. 1905-1915.

[27] S. RIPPA, An algorithm for selecting a good value for the parameter $c$ in radial basis function interpolation, Adv. Comput. Math., 11 (1999), pp. 193-210. 
[28] M. SCHEUERER, An alternative procedure for selecting a good value for the parameter $\mathrm{c}$ in refinterpolation, Adv. Comput. Math., 34 (2011), pp. 105-126.

[29] J. WANG AND G. LIU, A point interpolation meshless method based on radial basis functions, Int. J. Numer. Methods Eng., 54(11) (2002), pp. 1623-1648.

[30] J. WANG AND G. LIU, On the optimal shape parameters of radial basis func-tions used for $2 d$ meshless methods, Comput. Methods Appl. Mech. Eng., 191(23/24) (2002), pp. 2611-2630.

[31] G. LIU, G. ZHANG AND Y. GU, A meshfree radial point interpolation method (RPIM) for three dimensional solids, Comput. Mech., 36(6) (2005), pp. 421-430.

[32] L. LI, J. ZHU AND S. ZHANG, A hybrid radial boundary node method based on radial basis point interpolation, Eng. Anal. Boundary Elements, 33(11) (2009), pp. 1273-1283.

[33] L. DINIS, R. N. JORGE AND J. BELINHA, Analysis of $3 d$ solids using the natural neighbour radial point interpolation method, Comput. Methods Appl. Mech. Eng., 196(13-16) (2007), pp. 20092028.

[34] L. Dinis, R. N. Jorge AND J. Belinha, Analysis of plates and laminates using the natural neighbour radial point interpolation method, Eng. Anal. Boundary Elements, 32(3) (2008), pp. 267-279.

[35] G. R. LiU, K. Y. DAI AND T. T. NGUYen, A smoothed finite element method for mechanics problems, Comput. Mech., 39 (2007), pp. 859-877.

[36] K. Y. DAI AND G. R. LIU, Free and forced vibration analysis using the smoothed finite element method (SFEM), J. Sound Vib., 301 (2007), pp. 803-820.

[37] G. R. LIU, T. T. NGUYEN, K. Y. DAI AN D K. Y. LAM, Theoretical aspects of the smoothed finite element method (SFEM), Int. J. Numer. Methods Eng., 71(8) (2007), pp. 902-930.

[38] G. R. LIU AND S. S. QUEK, The Finite Element Method: A Practical Course, ButterworthHeinemann, 2013.

[39] G. R. LIU, A generalized gradient smoothing technique and the smoothed bilinear form for Galerkin formulation of a wide class of computational methods, Int. J. Comput. Methods, 5(2) (2008), pp. 199-236.

[40] G. R. LIU, W. ZENG AND H. NGUYEN-XUAN, Generalized stochastic cell-based smoothed finite element method (GS-CS-FEM) for solid mechanics, Finite Elements Anal. Design, 63 (2013), pp. 51-61.

[41] THOI. T. NGuYen, G. R. LiU, K. Y. LAM AND G. Y. ZHANG, A face-based smoothed finite element method (FS-FEM) for 3D linear and nonlinear solid mechanics problems using 4-node tetrahedral elements, Int. J. Numer. Methods Eng., 78 (2009), pp. 324-353.

[42] Z. C. He, G. R. LiU, Z. H. ZhONG, S. C. WU, G. Y. ZhANG AND A. G. ChenG, An edgebased smoothed finite element method (ES-FEM) for analyzing three-dimensional acoustic problems, Comput. Methods Appl. Mech. Eng., 199 (2009), pp. 20-33.

[43] G. R. LiU, T. NGUYEN-THOI, H. NGUYEN-XUAN AND K. Y. LAM, A node-based smoothed finite element method (NS-FEM) for upper bound solutions to solid mechanics problems, Comput. Struct., 87(12) (2009), pp. 14-26.

[44] H. WendLAND, Meshless Galerkin methods using radial basis functions, Math. Comput. American Mathematical Society, 68(228) (1999), pp. 1521-1531.

[45] Z. WU, Compactly supported positive definite radial functions, Adv. Comput. Math., 4 (1995), pp. 283-292.

[46] R. SCHABACK AND H. WENDLAND, Characterization and construction of radial basis functions, Multivariate Approximation and Applications, 2001.

[47] A. H. D. ChENG, M. A. GOlBerG, E. J. KANSA AND G. ZAMmito, Exponential convergence and $H_{c}$ Multiquadric collocation method for PDE, Num. Methods PDE, 19(5) (2003), pp. 571-594. 
[48] H. WENDLAND, Piecewise polynomial, positive definite and compactly supported radial functions of minimal degree, J. Adv. Comput. Math., 4 (1995), pp. 389-396.

[49] E. Ö̈ATE, F. Perazzo AND J. MiQuel, A finite point method for elasticity problems, Comput. Struct., 79(2225) (2001), pp. 2151-2163.

[50] E. Ö̈ATE, Derivation of stabilized equations for numerical solution of advective-diffusive transport and fluid flow problems, Comput. Methods Appl. Mech. Eng., 151(12) (1998), pp. 233-265.

[51] E. Ö̈Ate, S. Idelsohn, O. C. Zienkiewicz AND R. L. TAYlor, A finite point method in computational mechanics. applications to convective transport and fluid flow, Int. J. Numer. Meth. Eng., 39 (1996), pp. 3839-3866.

[52] T. J. LiszKA, C. A. M. DuARte And W. W. TwOrZydlo, $h \underline{p}$-meshless cloud method, Comput. Methods Appl. Mech. Eng., 139(14) (1996), pp. 263-288.

[53] G. R. LiU AND N. T. TrUNG, Smoothed Finite Element Method, Taylor and Francis, CRC Press, 2010.

[54] S. Timoshenko And J. GoodieR, Theory of Elasticity, McGraw-Hill, New York, 1970.

[55] G. R. LIU, Meshfree Methods: Moving Beyond the Finite Element Method, 2nd ed. Boca Raton: Taylor and Francis, CRC Press, 2002.

[56] T. J. LiszKA, C. A. M. DuARTE AND W. W. Tworzydlo, $h^{p}$-meshless cloud method, Comput. Methods Appl. Mech. Eng., 1399(1-4), pp. 263-288.

[57] Z. WU, Hermite-Birkhoff interpolation of scattered data by radial basis functions, Approximation Theory and Its Applications, 08-02 (1992), pp. 1000-9221.

[58] E. J. KANSA, Multiquadricsa scattered data approximation scheme with applications to computational fluid-dynamicsII solutions to parabolic, hyperbolic and elliptic partial differential equations, Comput. Math. Appl., 19(8) (1990), pp. 147-161.

[59] X. LIU, G. R. LIU, K. TAI AND K. Y. LAM, Radial point interpolation collocation method (RPICM) for partial differential equations, Comput. Math. Appl., 50(8-9) (2005), pp. 1425-1442.

[60] W. CHEN, Z. J. FU AND C. S. CHEN, Recent Advances on Radial Basis Function Collocation Methods, Springer Verlag, 2014.

[61] G. FASSHAUER, Meshfree Approximation Methods with MATLAB, Singapore Hackensack, N. J: World Scientific, 2007.

[62] R. D. GAUTHIER AND W. E. JAHSMAN, A quest for micropolar elastic constants, ASME. J. Appl. Mech., 42(2) (1975), pp. 369-374. 